\title{
Polypeptides Folding: Rules for the Calculation of the Backbone Dihedral Angles $\varphi$ starting from the Amino Acid Sequence
}

\section{Michele Larocca}

Submitted date: 19/03/2020 Posted date: 20/03/2020

Licence: CC BY 4.0

Citation information: Larocca, Michele (2020): Polypeptides Folding: Rules for the Calculation of the Backbone Dihedral Angles $\varphi$ starting from the Amino Acid Sequence. ChemRxiv. Preprint. https://doi.org/10.26434/chemrxiv.12000132.v2

Protein folding is strictly related to the determination of the backbone dihedral angles and depends on the information contained in the amino acid sequence as well as on the hydrophobic effect. To date, the type of information embedded in the amino acid sequence has not yet been revealed. The present study deals with these problematics and aims to furnish a possible explanation of the information contained in the amino acid sequence, showing and reporting rules to calculate the backbone dihedral angles $\varphi$. The study is based on the development of mechanical forces once specific chemical interactions are established among the side chain of the residues in a polypeptide chain. It aims to furnish a theoretical approach to predict backbone dihedral angles which, in the future, may be applied to computational developments focused on the prediction of polypeptide structures.

File list (2)

Polypeptides Folding Rules for the Calculation of the Ba... (611.00 KiB) view on ChemRxiv • download file 


\title{
Polypeptides Folding: Rules for the Calculation of the Backbone Dihedral Angles $\varphi$ starting from the Amino Acid Sequence
}

\author{
Michele Larocca \\ Planetario Osservatorio Astronomico di Anzi-Basilicata, via Salita Rosario s.n.c. 85010, Anzi \\ (PZ), Italy. \\ michele.larocca@studio.unibo.it \\ https://orcid.org/0000-0003-0613-5187
}

\begin{abstract}
The DNA binding domain of the $\gamma^{\delta}$ resolvase (PDB code: 1 res) is a peptide of 43 amino acids that was used in this study in order to describe a theoretical method useful to calculate the backbone dihedral angles $\varphi$ starting from the amino acid sequence. The
\end{abstract}

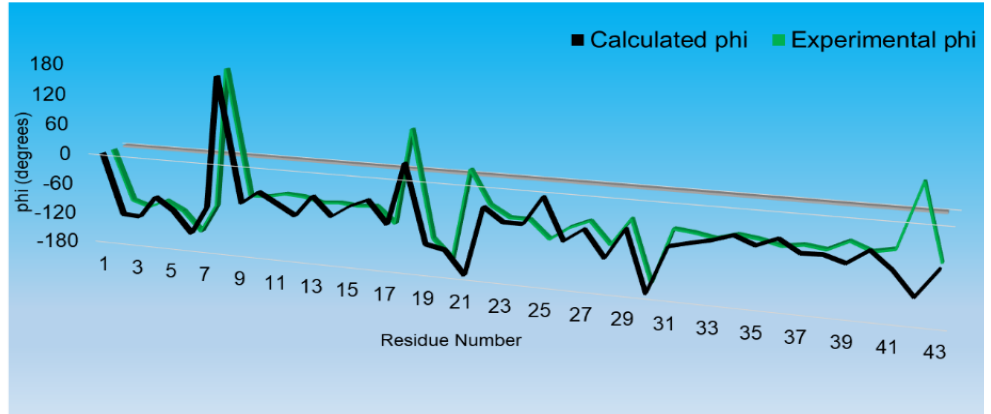
method is based on the development of mechanical forces due to the chemical interactions among the side chains of the residues, with the latter supposed to be the information required to fold the peptide. This study reports rules concerning the threshold distances related to the chemical interactions that occur and the univocal definition of the degrees of freedom (from now ahead twisting constants). With the rules described, the calculated backbone dihedral angles $\varphi$ of the DNA binding domain of the $\gamma^{\delta}$ resolvase, show a good agreement with the values experimentally determined, hence the methodology proposed, reveals to be a tool to calculate and predict at least the backbone dihedral angles $\varphi$.

\section{Introduction}

The prediction of a protein structure, starting from the amino acid sequence, turns to be very challenging and was deeply investigated over the years. Concerning this, in the past were reported very significant outcomes, as the thermodynamic hypothesis ${ }^{[1]}$ and the driving forces of the folding process ${ }^{[2-5]}$. In 2019 it was described a survey which focuses on the 
amino acid sequence to calculate the structure of two peptides (Leu-enkephalin and calcitonin ${ }^{[6]}$ and is based on the development of the main mechanical forces (MMFs) arising from the chemical interactions among the residues side chains. Anyway, concerning the calculations, the latter study showed some limits on the definition of the degrees of freedom $^{[6]}$ (now twisting constant) so that, this problem was further investigated in order to enunciate rules to define univocally the twisting constants and the threshold distances for the chemical interactions to occur. In this context, the present study realized on the DNA binding domain of the $\gamma^{\delta}$ resolvase (PDB code: 1 res) ${ }^{[7]}$, aims to establish rules that allow to predict and calculate the backbone dihedral angles $\varphi$, so that, combined with the previous study ${ }^{[6]}$, could furnish a theoretical method to predict the structure of peptides and proteins starting from the amino acid sequence. This study is focused on the chemical interactions developed amongst the side chains of the residues and these were considered to be the informations required to fold the polypeptide. The survey shows a good agreement between the calculated and the experimentally determined values, thus the method presented, combined to the previous ${ }^{[6]}$, reveals to be an efficient approach to predict at least the backbone dihedral angles $\varphi$ starting from the amino acid sequence.

\section{Methods}

\section{Measurement of distances}

The interatomic distances related to the chemical interactions were measured from a dedicated PDB file of the fully unfolded peptide (Figure 1). It was realized with Molefacture ${ }^{[8]}$ in $\mathrm{VMD}^{[9]}$.

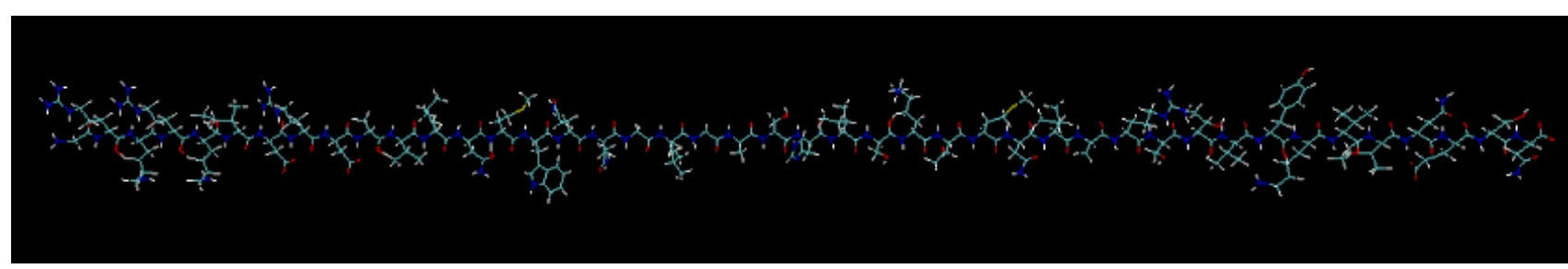

Figure 1. Fully unfolded peptide: the unfolded DNA binding domain of the $\gamma^{\delta}$ resolvase.

Rules for threshold distances and twisting constants $(\eta)$ for the calculation of the angles $\varphi$

For the calculations of the dihedral angles $\varphi$, is necessary to consider all the possible chemical interactions occurring among the side chains of the residues, according to the following rules, moreover the partial charges for the calculations were taken from the literature ${ }^{[10]}$ : 


\section{1) Electrostatic interaction between the terminal charges}

The electrostatic interaction between the terminal charges occurs to define a partial twisting, which is needed to calculate all the dihedral angles $\varphi$ of the peptide. For the polar residues, on the twisting constant it was found $\eta=2$, whereas $\eta$ have the specific values listed in Table 1 for the apolar residues.

\begin{tabular}{|c|c|}
\hline Residue (Apolar) & $\mathbf{n}$ \\
\hline Isoleucine & 0.1 \\
\hline Alanine & 15 \\
\hline Valine & 2 \\
\hline Leucine & 1.5 \\
\hline Methionine & 0.1 \\
\hline Glycine & -0.4 \\
\hline
\end{tabular}

Table 1. Twisting constants for the apolar residues used to calculate the folding factors.

2) Intrachain electrostatic interactions between charges (attraction and repulsion)

With the exception of the two terminal charges, the intrachain electrostatic interactions may occur up to a distance of $16 \AA$ for the attraction and $14.5 \AA$ for the repulsion. For the electrostatic attraction, it was found $\eta=3$, whereas $\eta=2$ in the case of the repulsion. For both attraction and repulsion, the final equilibrium distance is $r_{f}=2.9 \AA$.

3) Hydrophobic Interactions

For the polar residues, the hydrophobic interactions may occur up to a distance of $12 \AA$, whereas for the apolar residues, the threshold distances for the hydrophobic interactions are listed in table 2.

\begin{tabular}{|c|c|}
\hline Residue (Apolar) & Distance \\
\hline Isoleucine & $11.5 \AA$ \\
\hline Alanine & $11 \AA$ \\
\hline Valine & $13.5 \AA$ \\
\hline Leucine & $14 \AA$ \\
\hline Methionine & $12 \AA$ \\
\hline Glycine & $12 \AA$ \\
\hline
\end{tabular}

Table 2. Treshold distances required to establish hydrophobic interactions concerning apolar residues. 


\section{4) H-bonds}

$\mathrm{H}$-bonds may occur up to a distance of $14.6 \AA$ and the twisting constant for this type of interaction is $\eta=2$ for the calculation of $\varphi$. The final equilibrium distance is $r_{f}=2.9 \AA$.

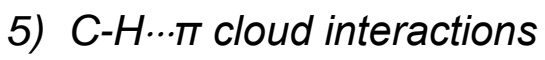

$\mathrm{C}-\mathrm{H} \cdots \mathrm{m}$ cloud interactions occur up to a distance of $14 \AA$ and the twisting constant is $\eta=1.5$ for the calculation of $\varphi$.

6) Pile stacking ( $\pi-\pi$ interactions)

In the molecule studied there are not $\pi-\pi$ interactions.

\section{Results and Discussion}

In this study, on the DNA binding domain of the $\gamma^{\delta}$ resolvase, has been reported a physicalchemical method to calculate the backbone dihedral angles $\varphi$ of a given polypeptide chain starting from the amino acid sequence. Calculations are based on the MMF method ${ }^{[6]}$ which focuses on the development of mechanical forces after the establishment of precise chemical interactions among the side chains of the residues. To define the occurring chemical interactions it was necessary, in turn, to define rules in order to calculate the partial twistings for each interaction occurred; then, for a given residue, all the partial twistings were added up in order to calculate the dihedral angle $\varphi$. The rules reported in the section Methods concern the twisting constants (former degrees of freedom ${ }^{[6]}$ ) and the distances for the chemical interactions to occur. Twisting constants have been reported to be fundamental for the determination of the folding factors ${ }^{[6]}$ and the latter to be, in turn, a fundamental physical measure for the calculation of dihedral angles. Anyway, in the previous study, the definition of the twisting constants was described as the main limit of the whole work $^{[6]}$. At this purpose, this work has been realized with the aim to define univocally the twisting constants in order to allow, in the end, the precise calculation of the backbone dihedral angles, specifically the backbone dihedral angles $\varphi$. With the rules proposed on the twisting constants, as well as on the threshold distances for the chemical interactions, the backbone dihedral angles $\varphi$ of 1 res have been calculated and then compared to the values experimentally obtained by $\mathrm{NMR}^{[7]}$. A comparison between the two sets of data is shown in Figure 2 and can be seen that 38 calculated values, out of 42 , show a good agreement with the experimental data ${ }^{[7]}$, whereas only 2 values have a weak agreement $\left(\varphi_{18}\right.$ and $\left.\varphi_{25}\right)$ and just 2 calculated values ( $\varphi_{21}$ and $\left.\varphi_{42}\right)$ do not match the experimental data. This results make the method proposed a good tool to determine and predict at least the backbone dihedral 
angles $\varphi$ of polypeptides, even if, further improvements will be realized studying other polypeptides.

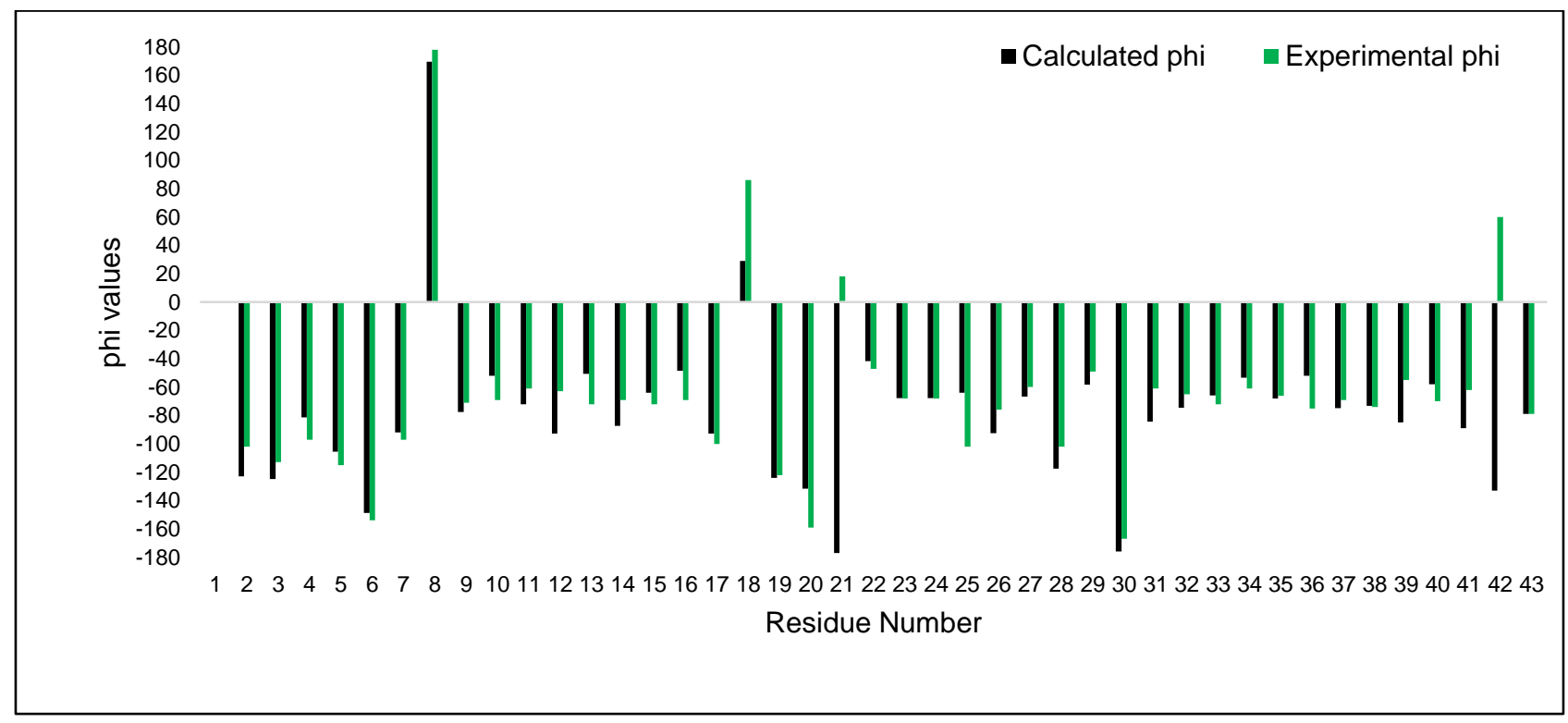

Figure 2. Histogram showing the comparison between calculated (black) and experimentally determined (green) backbone dihedral angles $\varphi$.

\section{Conclusions}

The present study deals with the problem of the structural prediction of polypeptides starting from the amino acid sequence. To date it is still a problem in the field of protein folding and still needs to be solved, although it has been deeply investigated over the years. To predict the structure of a protein, is necessary to determine the backbone dihedral angles $\varphi$ and $\psi$ and, as reported from previous studies, the informations to fold a polypeptide chain in its three dimensional structure are contained in the amino acid sequence. To date, the type of informations contained in the sequence still need to be revealed. In this regard, the present study aims to furnish a theoretical method that allows the calculation of backbone dihedral angles, specifically the dihedral angles $\varphi$, starting from the amino acid sequence, attempting to furnish a possible explanation of the informations to fold a polypeptide, according to the amino acid sequence. The chemical interactions among the side chains of the amino acid residues were supposed to be the informations required to fold the polypeptide: these allow the development of mechanical forces which induce the twistings necessary to fold the polypeptide. This approach, already reported in literature ${ }^{[6]}$ and deepen in this study, showed that it is possible to calculate the backbone dihedral angles $\varphi$ starting from the amino acid sequence. Results report that 38 values out of 42 (90\%) show a good agreement with the experimental data, 2 values show a weak agreement with the experimental data and only 2 
are wrong. The method thus, aims to be a useful tool to investigate partially the structure of a polypeptide chain (since concerns only $\varphi$ ), predicting it starting from the amino acid sequence. It may represent a good starting point for future computational developments that focus on the prediction of protein structures starting from the amino acid sequence.

\section{Bibliography}

[1] Anfinsen, C. B. (1973) Principles that govern the folding of protein chains. Science $181,223-230$.

[2] Dill, K. A. (1990) Dominant forces in protein folding. Biochemistry 29, 7133-7155.

[3] Camilloni, C., Bonetti, D., Morrone, A., Giri, R., Dobson, C. M., Brunori, M., Gianni, S. and Vendruscolo, M. (2016) Towards a structural biology of the hydrophobic effect in protein folding. Sci. Rep. 6, 28285.

[4] Baldwin, R. L., and Rose, G. D. (2016) How the hydrophobic factor drives protein folding. Proc. Natl. Acad. Sci. USA 113, 12462-12466.

[5] Kauzmann, W. (1964) The three dimensional structures of proteins. Biophys. J. 4, 4354.

[6] Larocca M., Foglia F. and Cilibrizzi A. (2019) Dihedral angle calculations to elucidate the folding of peptides through its main mechanical forces. Biochemistry 58, 8, 1032-1037.

[7] Liu T., DeRose E. F., and Mullen G. P. (1994) Determination of the structure of the DNA binding domain of $\gamma^{\delta}$ resolvase in solution. Protein Science, 3:1286-1295.

[8] Mayne, C. G., Saam, J., Schulten, K., Tajkhorshid, E. and Gumbart, J. C. (2013) Rapid parameterization of small molecules using the Force Field Toolkit. J. Comput. Chem. 34, 2757-2770.

[9] Humphrey, W., Dalke, A. and Schulten, K. (1996) VMD: Visual molecular dynamics. J. Molec. Graphics 14, 33-38.

[10] Cornell, W. D., Cieplak, P., Bayly, C. I., Gould, I. R., Merz, K. M. Jr., Ferguson, D. M., Spellmeyer, D. C., Fox, T., Caldwell, J. W. and Kollman, P. A. (1995) A Second Generation Force Field for the Simulation of Proteins, Nucleic Acids, and Organic Molecules. J. Am. Chem. Soc. 117, 5179-5197. 
Polypeptides Folding Rules for the Calculation of the Ba... (611.00 KiB) view on ChemRxiv • download file 
Supporting Information for

Polypeptides Folding: Rules for the Calculation of the Backbone Dihedral Angles $\varphi$ Starting from the Amino Acid Sequence

Michele Larocca

Planetario Osservatorio Astronomico di Anzi-Basilicata, via Salita Rosario s.n.c. 85010, Anzi (PZ), Italy.

michele.larocca@studio.unibo.it

https://orcid.org/0000-0003-0613-5187 
Calculations

\begin{tabular}{|c|c|c|c|}
\hline $\mathrm{K}\left(\mathrm{NA} \AA^{\wedge} 2 / \mathrm{c}^{\wedge} 2\right)$ & $1.12 \mathrm{E}+28$ & Note \\
\hline $\mathrm{e}(\mathrm{C})$ & $1.60 \mathrm{E}-19$ & q stands for a charge \\
\hline $\mathrm{SIN}\left(109.50^{\circ}\right)$ & 0.94 & or a partial charge \\
\hline & & & \\
\hline & & & \\
\hline & $\phi^{\circ}$ & & \\
\hline CALY1 & 0 & & \\
\hline & 0 & & \\
\hline
\end{tabular}

\begin{tabular}{|c|c|c|c|c|c|c|c|c|c|c|c|c|c|c|c|}
\hline \multicolumn{16}{|l|}{ ARG2 } \\
\hline & $\phi^{\circ}$ & \multirow{2}{*}{\multicolumn{2}{|c|}{$\phi^{\circ}$}} & & & & & & & & & & & & \\
\hline \multirow{15}{*}{$\begin{array}{l}\text { CALCULATED } \\
\text { EXPERIMENTAL }\end{array}$} & -123 & & & & & & & & & & & & & & \\
\hline & -102 & \multirow{2}{*}{\multicolumn{2}{|c|}{$\begin{array}{l}\text { Electrostatic Interaction } \\
\text { between terminal charges }\end{array}$}} & \multicolumn{2}{|c|}{ Hydrophobic interaction } & \multicolumn{2}{|c|}{ Electrostatic repulsion } & \multicolumn{2}{|c|}{ Electrostaticrepulsion } & \multicolumn{2}{|l|}{ Electrostatic repulsion } & \multicolumn{2}{|c|}{ Electrostaticrepulsion } & \multicolumn{2}{|c|}{ Hydrophobic interaction } \\
\hline & & & & \multirow[b]{2}{*}{ qGLY1:HA1 } & \multirow[b]{2}{*}{0.0698} & \multirow[b]{2}{*}{ qARG2:NH2 } & \multirow[b]{2}{*}{1} & \multirow{2}{*}{ qARG:NH2 } & & & & & & & \\
\hline & & qGIY:N & 1 & & & & & & 1 & qARG2:NH2 & 1 & qARG2:NH2 & 1 & qILE:HO1 & 0.0186 \\
\hline & & qASN43:0 & -1 & qARG2:CB & -0.0007 & qGLY1:N & 1 & qLYS3:NZ & 1 & qARG4:NH2 & 1 & qLYS5:NZ & 1 & qARG2:CB & -0.0007 \\
\hline & & ri & 154.93 & ri & 4.94 & $\mathrm{ri}$ & 7.74 & ri & 12.62 & ri & 7.26 & $\dot{n}$ & 15.08 & ri & 11.62 \\
\hline & & $\mathrm{F}$ & $-1.196-14$ & $\mathrm{~F}$ & $-5.745-16$ & $\mathrm{~F}$ & $4.796-12$ & $\mathrm{~F}$ & $1.800-12$ & $\mathrm{~F}$ & $5.44 E-12$ & $\mathrm{~F}$ & $1.266-12$ & $\mathrm{~F}$ & $-2.766-17$ \\
\hline & & U & $-1.85 E-12$ & U & $-2.84 E-15$ & u & $3.70 E-11$ & U & $2.27 E-11$ & U & $3.95 E-11$ & U & $1.90 E-11$ & U & $-3.21 E-16$ \\
\hline & & if & 2.9 & if & 2.9 & if & 2.9 & if & 2.9 & If & 2.9 & if & 2.9 & if & 2.9 \\
\hline & & $\theta$ & 17.12 & $\theta$ & 94.16 & $\theta$ & 17.12 & $\theta$ & 17.12 & $\theta$ & 17.12 & $\theta$ & 17.12 & $\theta$ & 10.46 \\
\hline & & $\mathrm{r}+\theta$ & 20.02 & $r+\theta$ & 97.06 & $f+\theta$ & 20.02 & $f+\theta$ & 20.02 & $f+\theta$ & 20.02 & $n+\theta$ & 20.02 & $f+\theta$ & 13.36 \\
\hline & & $\tau$ & $-2.395-13$ & $\tau$ & $-5.575-14$ & $\tau$ & $9.586-11$ & $\tau$ & $3.600-11$ & $\tau$ & $1.09 E-10$ & $\tau$ & $2.52 E-11$ & $\tau$ & $-3.695-16$ \\
\hline & & $\operatorname{tg}$ & 7.74 & $\operatorname{tg}$ & 0.05 & $\operatorname{tg}$ & 0.39 & $\operatorname{tg}$ & 0.63 & $\operatorname{tg}$ & 0.36 & $\operatorname{tg}$ & 0.75 & $\operatorname{tg}$ & 0.87 \\
\hline & & arctg & 1.44 & arctg & 0.05 & artg & 0.37 & arctg & 0.56 & arteg & 0.35 & artg & 0.65 & arctg & 0.72 \\
\hline & & degrees ${ }^{\circ}$ & 82.68 & degrees $^{\circ}$ & 2.92 & degrees $^{\circ}$ & 21.15 & degrees $^{\circ}$ & 32.24 & degrees $^{\circ}$ & 19.94 & degrees $^{\circ}$ & 37.01 & degrees $^{\circ}$ & 41.03 \\
\hline LYS3 & & & & & & & & & & & & & & & \\
\hline & $\phi^{\circ}$ & & & & & & & & & & & & & & \\
\hline CALCULATED & -125 & & $\phi^{\circ}$ & & & & & & & & & & & & \\
\hline EXPERIMENTAL & -113 & Electro & tatic Interacti & & lectrostatic re & epulsion & lectrostatic rep & ulsion & Electrost & tatic repulsion & Electrostat & ic repulsion & & Hydrophobic int & iteraction \\
\hline & & betwe & n terminal cha & & & & & & & & & & & & \\
\hline & & & GLY:N & 1 & qARG2:NH2 & 1 & qGLY1:N & 1 & & G4:NH2 & qLYS & 5:N2 & 1 & qLYS3:CB & -0.0094 \\
\hline & & & SNA3:O & -1 & qLYS3:NZ & 1 & qLYS3:NZ & 1 & & 153:N2 & qLYS & $3: \mathrm{Nz}$ & 1 & qlLE6:HG21 & 0.0236 \\
\hline & & & ri & 154.93 & ri & 12.62 & ri & 8 & & 14.02 & $n$ & i & 7.25 & $\mathrm{ri}$ & 12.65 \\
\hline & & & $\mathrm{F}$ & -1.19E-14 & $\mathrm{F}$ & $1.80 \mathrm{E}-12$ & $\mathrm{~F}$ & $4.48 E-12$ & & $1.46 E-12$ & $\mathrm{~F}$ & & $5.45 E-12$ & $\mathrm{~F}$ & $-3.977-16$ \\
\hline & & & u & $-1.85 E-12$ & $u$ & $2.27 E-11$ & u & $3.58 \mathrm{E}-11$ & & $2.05 E-11$ & $u$ & 3 & $3.95 E-11$ & u & $-5.03 E-15$ \\
\hline & & & If & 2.9 & If & 2.9 & If & 2.9 & & 2.9 & $\mathrm{r}$ & $f$ & 2.9 & if & 2.9 \\
\hline & & & $\theta$ & 17.12 & $\theta$ & 17.12 & $\theta$ & 17.12 & & 17.12 & $\theta$ & & 17.12 & $\theta$ & 10.46 \\
\hline & & & $f+\theta$ & 20.02 & $\mathrm{ff}+\theta$ & 20.02 & $\mathrm{ff}+\theta$ & 20.02 & & 20.02 & $n t$ & $+\theta$ & 20.02 & $f+\theta$ & 13.36 \\
\hline & & & $\tau$ & $-2.39 E-13$ & $\tau$ & $3.60 \mathrm{E}-11$ & $\tau$ & $8.976-11$ & & $2.92 E-11$ & $t$ & 1 & 1.09E-10 & $\tau$ & $-5.31 E-15$ \\
\hline & & & tg & 7.74 & $\operatorname{tg}$ & 0.63 & tg & 0.40 & & 0.70 & t & $g$ & 0.36 & tg & 0.95 \\
\hline & & & artg & 1.44 & artg & 0.56 & artg & 0.38 & & 0.61 & arc & & 0.35 & artg & 0.76 \\
\hline & & & grees ${ }^{\circ}$ & 82.68 & degrees $^{\circ}$ & 32.24 & degrees $^{\circ}$ & 21.79 & & 35.02 & degr & ees ${ }^{\circ}$ & 19.92 & degrees $^{\circ}$ & 43.45 \\
\hline ARG4 & & & & & & & & & & & & & & & \\
\hline & $\phi^{\circ}$ & & & & & & & & & & & & & & \\
\hline CALCULATED & .81 & $\phi^{\circ}$ & & & & & & & & & & & & & \\
\hline EXPERRMENTAL & .97 & Electrostatic Inter & & Electrostatic rep & pulsion & Electrostaticrepul & & Hydrophobicinte & action & Electrostatic Attractio & & Electrostaticre & & Electrostaticrep & oulsion \\
\hline & & between termina & charges & & & & & & & & & & & & \\
\hline & & qGLYY:N & 1 & qARG4:NH2 & 1 & qARG4:NH2 & 1 & qARG4:CB & -0.0007 & qARG4:NH2 & 1 & qARG4:NH2 & 1 & qARG4:NH2 & 1 \\
\hline & & qASNA3:0 & -1 & qLYS3:N2 & 1 & qLYS5:NZ & 1 & ql|lE:HG21 & 0.0236 & qASP7:002 & -1 & qARG8:NH2 & 1 & qGIY1:N & 1 \\
\hline & & ri & 154.93 & ri & 14.02 & ri & 12.62 & ri & 7.91 & $\mathrm{ri}$ & 15.7 & ri & 14.52 & ri & 13.14 \\
\hline & & $\mathrm{F}$ & $-1.195-14$ & $\mathrm{~F}$ & $1.46 E-12$ & $\mathrm{~F}$ & $1.80 E-12$ & $\mathrm{~F}$ & $-7.576-17$ & $\mathrm{~F}$ & $-1.16 E-12$ & $\mathrm{~F}$ & $1.366-12$ & $\mathrm{~F}$ & $1.666-12$ \\
\hline & & U & $-1.85 E-12$ & u & $2.05 E-11$ & u & $2.27 E-11$ & U & $-5.995-16$ & U & $-1.83 E-11$ & U & $1.97 E-11$ & U & $2.186-11$ \\
\hline & & if & 2.9 & if & 2.9 & If & 2.9 & if & 2.9 & if & 2.9 & if & 2.9 & if & 2.9 \\
\hline & & $\theta$ & 17.12 & $\theta$ & 17.12 & $\theta$ & 17.12 & $\theta$ & 10.46 & $\theta$ & 25.68 & $\theta$ & 17.12 & $\theta$ & 17.12 \\
\hline & & $\mathrm{r}+\theta$ & 20.02 & $r+\theta$ & 20.02 & $\mathrm{r}+\theta$ & 20.02 & $\mathrm{ft}+\theta$ & 13.36 & $\mathrm{r}+\theta$ & 28.58 & $\mathrm{r}+\theta$ & 20.02 & $\mathrm{If}+\theta$ & 20.02 \\
\hline & & $\tau$ & $-2.395-13$ & $\tau$ & $2.92 E-11$ & $\tau$ & $3.60 E-11$ & $\tau$ & $-1.01 E-15$ & $\tau$ & $-3.325-11$ & $\tau$ & $2.725-11$ & $\tau$ & $3.32 E-11$ \\
\hline & & $\operatorname{tg}$ & 7.74 & $\operatorname{tg}$ & 0.70 & $\operatorname{tg}$ & 0.63 & $\operatorname{tg}$ & 0.59 & $\operatorname{tg}$ & 0.55 & $\operatorname{tg}$ & 0.73 & $\operatorname{tg}$ & 0.66 \\
\hline & & arttg & 1.44 & artg & 0.61 & artg & 0.56 & artg & 0.53 & artg & 0.50 & artg & 0.63 & arttg & 0.58 \\
\hline & & degrees & 82.68 & degrees & 35.02 & degrees ${ }^{\circ}$ & 32.24 & degrees & 30.64 & degrees ${ }^{\circ}$ & 28.80 & degrees & 35.97 & degrees" & 33.30 \\
\hline
\end{tabular}




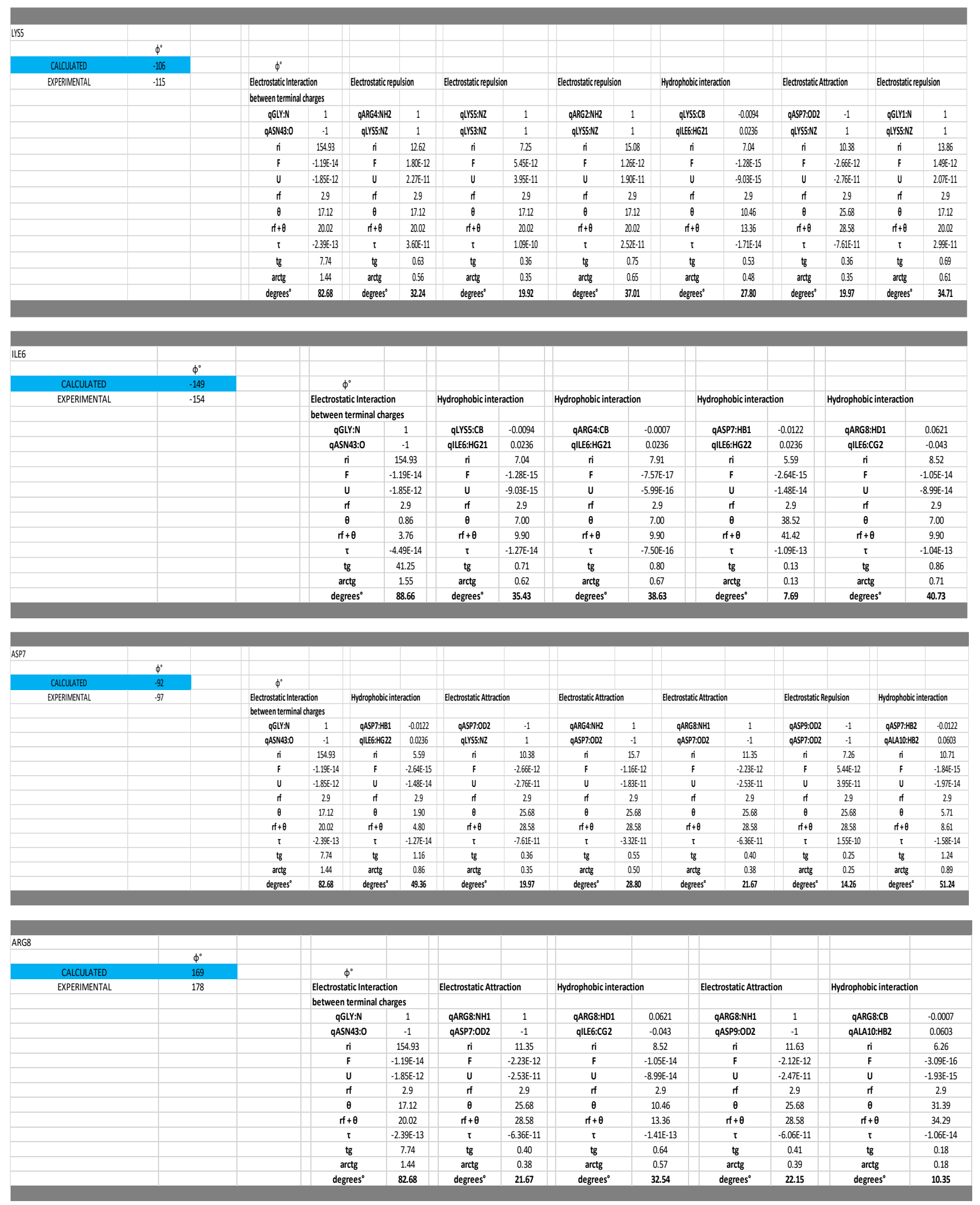



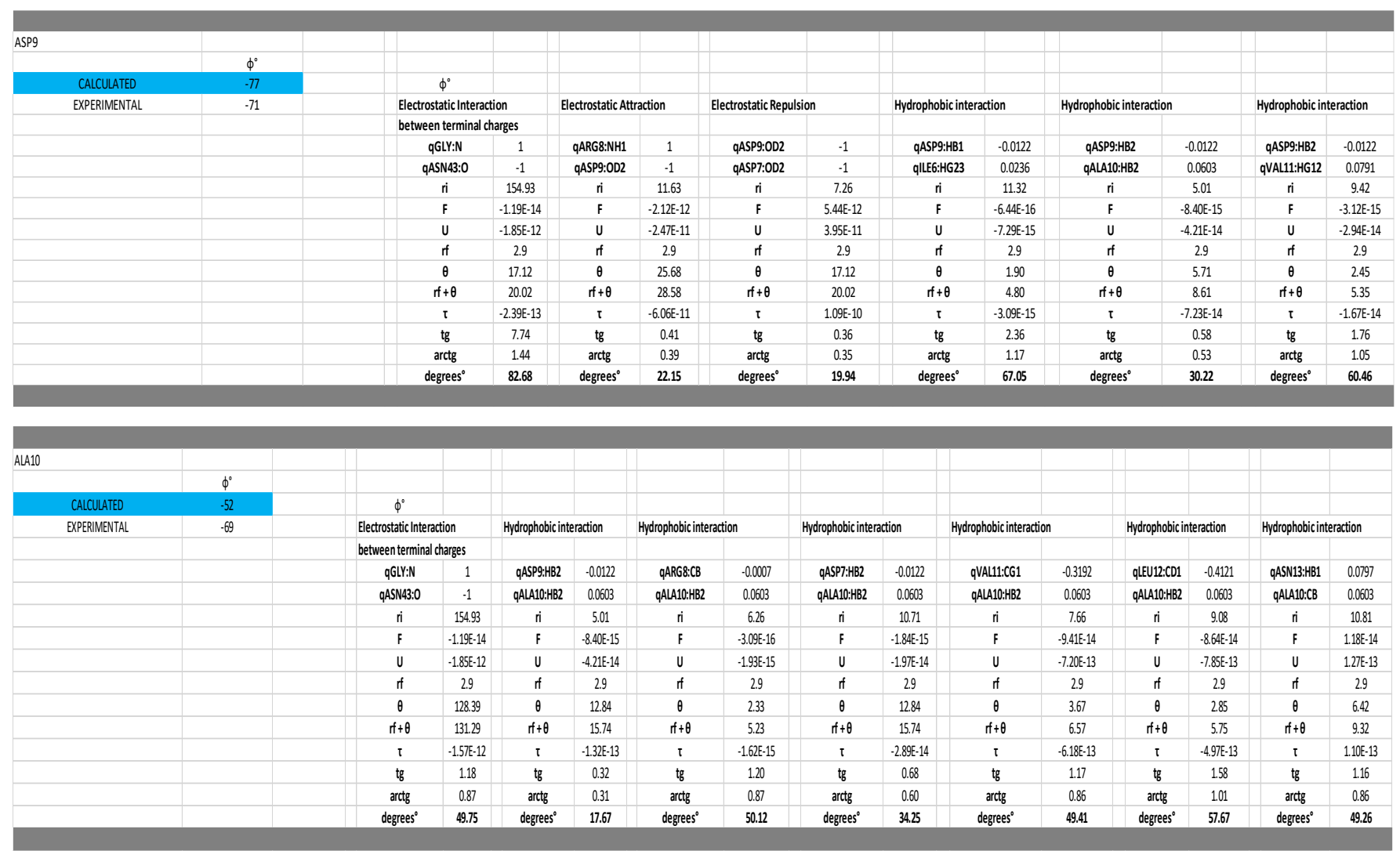

\begin{tabular}{|c|c|c|c|c|c|c|c|c|c|c|c|c|c|c|c|}
\hline \multicolumn{16}{|l|}{ VAL11 } \\
\hline & $\phi^{\circ}$ & & & & & & & & & & & & & & \\
\hline \multirow{15}{*}{$\begin{array}{l}\text { CALCULATED } \\
\text { EXPERIMENTAL }\end{array}$} & .72 & $\phi^{\circ}$ & & & & & & & & & & & & & \\
\hline & -61 & Electrostatic Inter & & \multicolumn{2}{|c|}{ Hydrophobicinteraction } & \multicolumn{2}{|c|}{ Hydrophobic interaction } & \multicolumn{2}{|c|}{ Hydrophobic interaction } & \multicolumn{2}{|c|}{ Hydrophobic interaction } & \multicolumn{2}{|c|}{ Hydrophobic interaction } & \multicolumn{2}{|c|}{ Hydrophobic interaction } \\
\hline & & \multicolumn{2}{|c|}{ between terminal charges } & & & & & & & & & & & & \\
\hline & & qGLYY:N & 1 & qVAL11:CG1 & -0.3192 & qASP9:HB2 & -0.0122 & qVAL11:CG2 & -0.3192 & qVAL11:CG1 & -0.3192 & qVAL11:CG2 & -0.3192 & qVAL11:CG2 & -0.3192 \\
\hline & & qASNA3:0 & -1 & qALA10:HB2 & 0.0603 & qVAL11:HG12 & 0.0791 & qLEU12:HD21 & 0.1 & qASN13:HB1 & 0.0797 & qMET14:HB1 & 0.0241 & qTRP15:HB1 & 0.0339 \\
\hline & & ri & 154.93 & ri & 7.66 & ri & 9.42 & ri & 9.79 & ri & 5.04 & ri & 10.52 & ri & 12.26 \\
\hline & & $\mathrm{F}$ & $-1.195-14$ & $\mathrm{~F}$ & $-9.41 E-14$ & $\mathrm{~F}$ & $-3.126-15$ & $\mathrm{~F}$ & $-9.555-14$ & $\mathrm{~F}$ & $-2.875-13$ & $\mathrm{~F}$ & $-1.995-14$ & $\mathrm{~F}$ & $-2.066-14$ \\
\hline & & u & $-1.855-12$ & u & $-7.20 \mathrm{E}-13$ & u & $-2.945-14$ & u & $-9.355-13$ & u & $-1.45 E-12$ & u & $-2.106-13$ & u & $-2.53 E-13$ \\
\hline & & if & 2.9 & if & 2.9 & If & 2.9 & if & 2.9 & if & 2.9 & if & 2.9 & if & 2.9 \\
\hline & & $\theta$ & 17.12 & $\theta$ & 19.97 & $\theta$ & 29.96 & $\theta$ & 6.66 & $\theta$ & 4.89 & $\theta$ & 8.56 & $\theta$ & 7.49 \\
\hline & & $r+\theta$ & 20.02 & $n+\theta$ & 22.87 & $\mathrm{r}+\theta$ & 32.86 & $r+\theta$ & 9.56 & $\mathrm{r}+\theta$ & 7.79 & $\mathrm{f}+\theta$ & 11.46 & $n+\theta$ & 10.39 \\
\hline & & $\mathrm{\tau}$ & $-2.395-13$ & $\mathrm{t}$ & $-2.155-12$ & $\tau$ & $-1.02 E-13$ & $\mathrm{t}$ & $-9.135-13$ & $\tau$ & $-2.246-12$ & $\tau$ & $-2.286-13$ & $\tau$ & $-2.14 E-13$ \\
\hline & & $\operatorname{tg}$ & 7.74 & $\operatorname{tg}$ & 0.33 & $\operatorname{tg}$ & 0.29 & $\operatorname{tg}$ & 1.02 & $\operatorname{tg}$ & 0.65 & $\operatorname{tg}$ & 0.92 & $\operatorname{tg}$ & 1.18 \\
\hline & & arctg & 1.44 & artg & 0.32 & arttg & 0.28 & artg & 0.80 & artg & 0.57 & artg & 0.74 & arctg & 0.87 \\
\hline & & degrees $^{\circ}$ & 82.68 & degrees ${ }^{\circ}$ & 18.53 & degrees & 16.00 & degrees $^{\circ}$ & 45.71 & degrees & 32.91 & degrees $^{\circ}$ & 42.57 & degrees ${ }^{\circ}$ & 49.75 \\
\hline
\end{tabular}

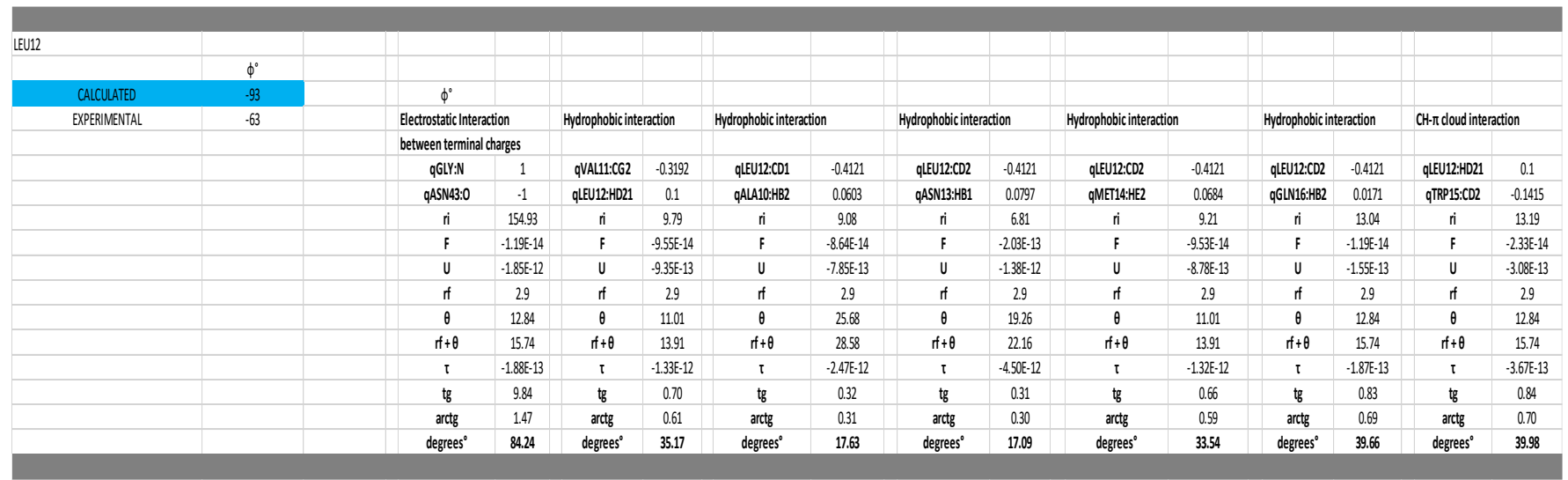




\begin{tabular}{|c|c|c|c|c|c|c|c|c|c|c|c|c|c|c|c|c|c|}
\hline \multicolumn{18}{|l|}{ ASN13 } \\
\hline & $\phi^{\circ}$ & & & & & & & & & & & & & & & & \\
\hline \multirow{13}{*}{$\begin{array}{l}\text { CALCUATAED } \\
\text { EXPERRMENTAL }\end{array}$} & .51 & $\phi^{\circ}$ & & & & & & & & & & & & & & & \\
\hline & .72 & Electrostatic Inter & & \multicolumn{2}{|c|}{ Hydrophobicinteraction } & \multicolumn{2}{|c|}{ Hydrophobicinteraction } & \multicolumn{2}{|c|}{ Hydrophobicinteraction } & H-bond & & H.bond & & H-bond & & H.bond & \\
\hline & & $q G I Y: N$ & 1 & qEVU12:02 & .0 .4121 & qVAll1:CG1 & .0 .3192 & qASN13:HB1 & 0.0797 & qMET14:50 & 0.2737 & qTRP15:HE1 & 0.3412 & qGIN16:HE22 & 0.4251 & qGL1N17:HE22 & 0.4251 \\
\hline & & gASNA3:O & -1 & gASN13:HB1 & 0.0797 & QASN13:HB1 & 0.0797 & qALA10:CB & 0.0603 & qASN13:H022 & 0.4196 & QASN13:ND2 & .0 .9191 & GASN13:ND2 & -0.9191 & qASN13:ND2 & .0 .9191 \\
\hline & & ri & 154.93 & ri & 6.81 & i & 5.04 & if & 10.81 & ri & 9.59 & i & 6.3 & ri & 13.16 & i & 13.89 \\
\hline & & $\mathrm{F}$ & $-1.196-14$ & $\mathrm{~F}$ & $-2035-13$ & $\mathrm{~F}$ & $-2.877-13$ & $\mathrm{~F}$ & $1.18 E-14$ & $\mathrm{~F}$ & $3.586-13$ & $\mathrm{~F}$ & $-2.277-12$ & $\mathrm{~F}$ & $-6.47 F-13$ & $\mathrm{~F}$ & $-5.81 E-13$ \\
\hline & & if & 2.9 & if & 2.9 & if & 2.9 & if & 2.9 & if & 2.9 & if & 2.9 & if & 2.9 & if & 2.9 \\
\hline & & $\theta$ & 17.12 & $\theta$ & 3.80 & $\theta$ & 4.89 & $\theta$ & 11.41 & $\theta$ & 17.12 & $\theta$ & 17.12 & $\theta$ & 17.12 & $\theta$ & 17.12 \\
\hline & & $n+\theta$ & 20.02 & $n+\theta$ & 6.70 & $\mathrm{f}+\theta$ & 7.79 & $f+\theta$ & 14.31 & $f+\theta$ & 20.02 & $\mathrm{f}+\theta$ & 20.02 & $n+\theta$ & 20.02 & $n+\theta$ & 20.02 \\
\hline & & $\tau$ & $-2.395-13$ & $\tau$ & $-1.366-12$ & $\tau$ & $-2.245-12$ & $\tau$ & $1.699-13$ & $\tau$ & $7.177-12$ & $\tau$ & $-4.545-11$ & ז & $-1.296-11$ & $\tau$ & -1.166-11 \\
\hline & & $\operatorname{tg}$ & 7.74 & $\operatorname{tg}$ & 1.02 & $\operatorname{tg}$ & 0.65 & $\operatorname{tg}$ & 0.76 & $\operatorname{tg}$ & 0.48 & $\operatorname{tg}$ & 0.31 & $\operatorname{tg}$ & 0.66 & $\operatorname{tg}$ & 0.69 \\
\hline & & artg & 1.44 & artg & 0.79 & artg & 0.57 & arctg & 0.65 & arctg & 0.45 & artg & 0.30 & arctg & 0.58 & artg & 0.61 \\
\hline & & degreess & 82.68 & degrees ${ }^{\circ}$ & 45.47 & degrees ${ }^{\circ}$ & 32.91 & degrees & 37.08 & degrees & 25.61 & degrees ${ }^{\circ}$ & 17.48 & degrees & 33.34 & degrees ${ }^{\circ}$ & 34.7 \\
\hline WVIEII4 & $\phi^{\circ}$ & & & & & & & & & & & & & & & & \\
\hline CALCULATED & -87 & & $\phi^{\circ}$ & & & & & & & & & & & & & & \\
\hline EXPERIMENTAL & -69 & Electros & atic Interactio & & I-bond & & drophobic inter & action & Hydrophob & icinteraction & H-bond & & & H-bond & & H-bond & \\
\hline & & betwee & terminal cha & & & & & & & & & & & & & & \\
\hline & & & ilY:N & 1 & qMET14:SD & 0.2737 & qLEU12:CD2 & -0.4121 & qVAL11 & .0 .3192 & qMET1 & SD & 2737 & qMET14:SD & 0.2737 & qMET14:SD & 0.2737 \\
\hline & & & NA3:0 & -1 & qASN13:HD22 & 0.4196 & qMET14:HE2 & 0.0684 & qMET14 & 0.0241 & qGLN16. & AE22 & 1251 & qTRP15:HE1 & 0.3412 & qGIN17:HE21 & 0.4251 \\
\hline & & & ri & 154.93 & ri & 9.59 & ri & 9.21 & $\mathrm{ri}$ & 10.52 & in & 6 & 32 & ri & 9.08 & ri & 11.48 \\
\hline & & & $F$ & $-1.19 E-14$ & $\mathrm{~F}$ & $3.58 E-13$ & $\mathrm{~F}$ & $-9.53 E-14$ & $\mathrm{~F}$ & $-1.996-14$ & $\mathrm{~F}$ & 8.35 & $6 E-13$ & $\mathrm{~F}$ & $3.25 E-13$ & $\mathrm{~F}$ & $2.53 E-13$ \\
\hline & & & U & $-1.855-12$ & U & $3.43 E-12$ & U & $-8.78 E-13$ & u & $-2.100-13$ & u & 5.28 & BE-12 & U & $2.95 E-12$ & U & $2.91 E-12$ \\
\hline & & & If & 2.9 & If & 2.9 & If & 2.9 & if & 2.9 & if & 2 & 9 & If & 2.9 & If & 2.9 \\
\hline & & & $\theta$ & 0.86 & $\theta$ & 17.12 & $\theta$ & 6.66 & $\theta$ & 8.56 & $\theta$ & 17 & 12 & $\theta$ & 17.12 & $\theta$ & 17.12 \\
\hline & & & $f+\theta$ & 3.76 & $n+\theta$ & 20.02 & $\mathrm{ft}+\theta$ & 9.56 & $n+$ & 11.46 & $\mathrm{rt}+$ & 20 & 0.02 & $f+\theta$ & 20.02 & $f+\theta$ & 20.02 \\
\hline & & & $\tau$ & $-4.49 E-14$ & $\tau$ & $7.17 E-12$ & $\tau$ & $-9.11 E-13$ & $\mathrm{t}$ & $-2.28 E-13$ & $\mathrm{t}$ & 1.67 & 7E-11 & $\tau$ & $6.50 E-12$ & $\tau$ & $5.07 E-12$ \\
\hline & & & $\operatorname{tg}$ & 41.25 & $\operatorname{tg}$ & 0.48 & tg & 0.96 & tg & 0.92 & tg & 0 & 32 & $\operatorname{tg}$ & 0.45 & $\operatorname{tg}$ & 0.57 \\
\hline & & & rctg & 1.55 & artg & 0.45 & arctg & 0.77 & artt & 0.74 & art & 0 & 31 & artg & 0.43 & artg & 0.52 \\
\hline & & & rrees $^{\circ}$ & 88.66 & degrees $^{\circ}$ & 25.61 & degrees $^{\circ}$ & 43.96 & degre & 42.57 & degre & 17 & 1.53 & degrees $^{\circ}$ & 24.41 & degrees ${ }^{\circ}$ & 29.85 \\
\hline & $\phi^{\circ}$ & & & & & & & & & & & & & & & & \\
\hline CALCULATED & .64 & $\phi^{\circ}$ & & & & & & & & & & & & & & & \\
\hline EXPERMENTAL & .72 & Electrostatic Intera & & H.bond & & H.bond & & CH-rcloud interatt & & Hydrophobic interatect & & H.bond & & H.bond & & CH-r cloud intera & \\
\hline & & between terminal & charges & & & & & & & & & & & & & & \\
\hline & & gGIY:N & 1 & GMET14:5D & 0.2737 & qTRP15:HE1 & 0.3412 & qLEU12:H021 & 0.1 & qVAl11:CG2 & .03192 & qTRP15:NE1 & .03418 & qTRP15:NE1 & .03418 & qGIY18:HA1 & 0.0698 \\
\hline & & gASNA3:O & -1 & qTRP15:HE1 & 0.3412 & qASN13:ND2 & .09191 & qTRP15:CD2 & -0.1415 & qTRP15:HB1 & 0.0339 & qGIN16:HE21 & 0.4251 & GGIN17:HE22 & 0.4251 & qTRP15:C02 & .0 .1415 \\
\hline & & ii & 154.93 & ii & 9.08 & ri & 6.3 & ri & 13.19 & ri & 12.26 & i & 10.79 & ri & 9.14 & ri & 10.7 \\
\hline & & $\mathrm{F}$ & $-1.196-14$ & $\mathrm{~F}$ & $3.25 E-13$ & $\mathrm{~F}$ & $-2.276-12$ & $\mathrm{~F}$ & $-2.33 E-14$ & $\mathrm{~F}$ & $-2.066-14$ & $\mathrm{~F}$ & $-3.586-13$ & $\mathrm{~F}$ & $-4.996-13$ & $\mathrm{f}$ & $-2.47-14$ \\
\hline & & u & $-1.855-12$ & U & $2.955-12$ & U & $-1.435-11$ & u & $-3.086-13$ & U & $-2535-13$ & u & $-3.866-12$ & U & $-4.566-12$ & U & $-2.65-13$ \\
\hline & & If & 2.9 & if & 2.9 & If & 2.9 & If & 2.9 & If & 2.9 & if & 2.9 & If & 2.9 & if & 2.9 \\
\hline & & $\theta$ & 17.12 & $\theta$ & 17.12 & $\theta$ & 17.12 & $\theta$ & 12.84 & $\theta$ & 9.78 & $\theta$ & 17.12 & $\theta$ & 17.12 & $\theta$ & 12.84 \\
\hline & & $\mathrm{n}+\theta$ & 20.02 & $f+\theta$ & 20.02 & $\mathrm{r}+\theta$ & 20.02 & $\mathrm{r}+\theta$ & 15.74 & $\mathrm{r}+\theta$ & 12.68 & $\mathrm{nt}+\theta$ & 20.02 & $n+\theta$ & 20.02 & $\mathrm{n}+\theta$ & 15.74 \\
\hline & & $\tau$ & $-2.395-13$ & $\tau$ & $6.50 E-12$ & $\tau$ & $-4.546-11$ & $\tau$ & $-3.677-13$ & $\tau$ & $-2.625-13$ & $\tau$ & $-7.166-12$ & $\tau$ & $-9.986-12$ & $\tau$ & $-3.895-13$ \\
\hline & & tg & 7.74 & $\operatorname{tg}$ & 0.45 & $\operatorname{tg}$ & 0.31 & tg & 0.84 & tg & 0.97 & $\operatorname{tg}$ & 0.54 & $\operatorname{tg}$ & 0.46 & $\operatorname{tg}$ & 0.68 \\
\hline & & artg & 1.4 & artg & 0.43 & artg & 0.30 & artg & 0.70 & artg & 0.77 & artig & 0.49 & artg & 0.43 & artg & 0.60 \\
\hline & & degrees" & 82.68 & degrees" & 24.41 & degreess & 17.48 & degrees ${ }^{\circ}$ & 39.98 & degrees ${ }^{\circ}$ & 4.05 & degrees ${ }^{\circ}$ & 28.34 & degrees" & 24.55 & degreess & 34.23 \\
\hline GIN16 & & & & & & & & & & & & & & & & & \\
\hline & $\phi^{\circ}$ & & & & & & & & & & & & & & & & \\
\hline CALCULATED & .48 & $\phi^{\circ}$ & & & & & & & & & & & & & & & \\
\hline EXPERMENTAL & .69 & Electrostatic Inter & & H.bond & & H-bond & & H.bond & & Hydrophobicinteract & & H.bond & & Hydrophobicinte & teraction & Hydrophobicinter & ation \\
\hline & & between teminal & charges & & & & & & & & & & & & & & \\
\hline & & qGLYY:N & 1 & qTRP15:NE1 & .0 .3418 & qMEETA:SO & 0.2737 & qGIN16:HE22 & 0.4251 & qIEU12:CO2 & .0 .421 & qGIN17:HE21 & 0.4251 & qGIY18:HA1 & 0.0698 & qlEU19:H012 & 0.1 \\
\hline & & gASN43:0 & -1 & GGLN16:HE2I & 0.4251 & qGIN16:HE2Z & 0.4251 & gASN13:ND2 & .0 .9191 & gGIN16:HB2 & 0.0171 & qGL116:NE2 & .0 .9407 & qGIN16:CB & -0.0036 & qGIN16:CB & .00036 \\
\hline & & i & 154.93 & i & 10.79 & i & 6.32 & ri & 13.16 & ri & 13.04 & กं & 11.92 & i & 7.26 & กं & 12.11 \\
\hline & & $\mathrm{F}$ & $-1.196-14$ & $\mathrm{~F}$ & $-3.586-13$ & $\mathrm{~F}$ & $8.355-13$ & $\mathrm{~F}$ & $-6.47-13$ & $\mathrm{~F}$ & $-1.195-14$ & $\mathrm{~F}$ & $-8.076-13$ & $\mathrm{~F}$ & $-1.377-15$ & $\mathrm{~F}$ & $.7 .045-16$ \\
\hline & & U & $-1.85 E-12$ & U & $-3.866-12$ & U & $5.286-12$ & U & $.851 E-12$ & U & $-1.555-13$ & U & $-9.62 E-12$ & U & $.9 .92 E-15$ & U & $-8.52 E-15$ \\
\hline & & If & 2.9 & if & 2.9 & If & 2.9 & If & 2.9 & If & 2.9 & If & 2.9 & ff & 2.9 & If & 2.9 \\
\hline & & $\theta$ & 17.12 & $\theta$ & 17.12 & $\theta$ & 17.12 & $\theta$ & 17.12 & $\theta$ & 5.71 & $\theta$ & 17.12 & $\theta$ & 51.36 & $\theta$ & 5.71 \\
\hline & & $\mathrm{I}+\theta$ & 20.02 & $\mathrm{n}+\theta$ & 20.02 & $\mathrm{r}+\theta$ & 20.02 & $f+\theta$ & 20.02 & $f+\theta$ & 8.61 & $\mathrm{n}+\mathrm{\theta}$ & 20.02 & $\mathrm{ft}+\theta$ & 54.26 & $\mathrm{ft}+\theta$ & 8.61 \\
\hline & & $\tau$ & $-2.39 E-13$ & $\tau$ & $-7.166-12$ & $\tau$ & $1.67-11$ & $\tau$ & $-1.29 E-11$ & $\mathrm{t}$ & $-1.02 E-13$ & $\tau$ & $-1.62 E-11$ & $\tau$ & $-7.42 E-14$ & $\mathrm{t}$ & $-6.066-15$ \\
\hline & & $\operatorname{tg}$ & 7.74 & $\operatorname{tg}$ & 0.54 & $\operatorname{tg}$ & 0.32 & $\operatorname{tg}$ & 0.66 & $\operatorname{tg}$ & 1.52 & $\operatorname{tg}$ & 0.60 & $\operatorname{tg}$ & 0.13 & $\operatorname{tg}$ & 1.41 \\
\hline & & artg & 1.4 & artg & 0.49 & artg & 0.31 & arctg & 0.58 & artg & 0.99 & artg & 0.54 & arctg & 0.13 & artg & 0.95 \\
\hline & & degrees ${ }^{\circ}$ & 82.68 & degreess & 28.34 & degrees ${ }^{\circ}$ & 17.53 & degrees & 33.34 & degrees" & 56.60 & degrees & 30.79 & degrees & 7.63 & degreess & 54.63 \\
\hline
\end{tabular}



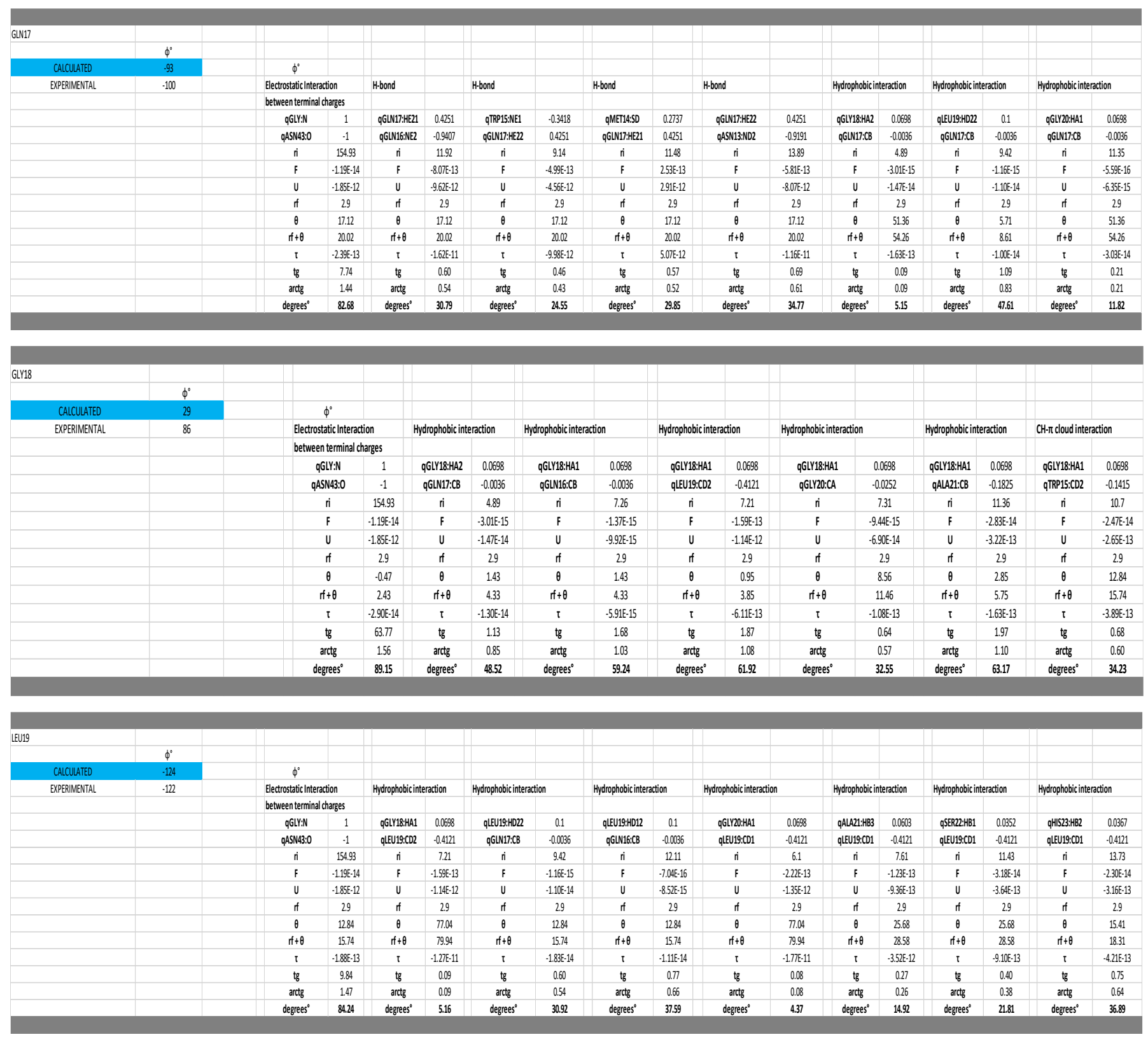

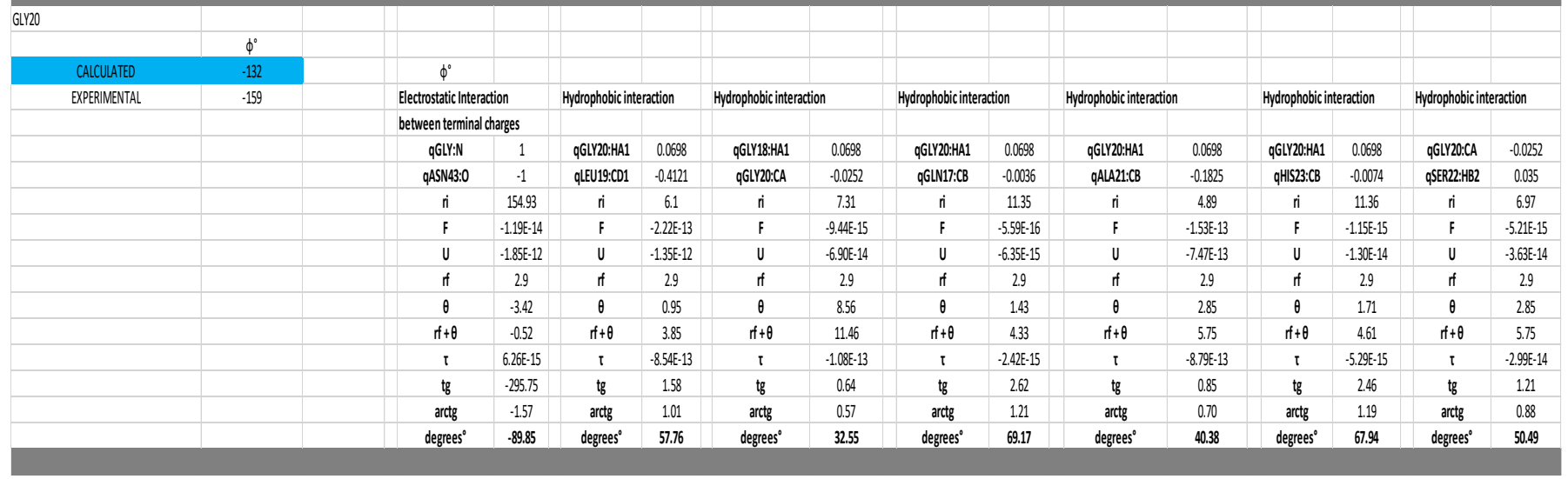




\begin{tabular}{|c|c|c|c|c|c|c|c|c|c|c|c|}
\hline \multicolumn{12}{|l|}{ ALA21 } \\
\hline & $\phi^{\circ}$ & & & & & & & & & & \\
\hline \multirow{15}{*}{ EXPERIMENTAL } & -177 & $\phi^{\circ}$ & & & & & & & & & \\
\hline & 18 & \multirow{2}{*}{\multicolumn{2}{|c|}{$\begin{array}{l}\text { Electrostatic Interaction } \\
\text { between terminal charges }\end{array}$}} & \multicolumn{2}{|c|}{ Hydrophobic interaction } & \multicolumn{2}{|c|}{ Hydrophobic interaction } & \multicolumn{2}{|c|}{ Hydrophobic interaction } & \multicolumn{2}{|c|}{ Hydrophobic interaction } \\
\hline & & & & & & & & & & & \\
\hline & & qGLY:N & 1 & qGLY20:HA1 & 0.0698 & qALA21:HB3 & 0.0603 & gSER22:HB1 & 0.035 & gHIS23:HB1 & 0.0367 \\
\hline & & qASN43:0 & -1 & gALA21:CB & -0.1825 & qLEU19:CD1 & -0.4121 & gALA21:CB & -0.1825 & GALA21:CB & -0.1825 \\
\hline & & ri & 154.93 & ri & 4.89 & ri & 7.61 & ri & 5.25 & ri & 8.32 \\
\hline & & $F$ & $-1.19 E-14$ & $\mathrm{~F}$ & $-1.53 E-13$ & $\mathrm{~F}$ & $-1.23 \mathrm{E}-13$ & $\mathrm{~F}$ & $-6.64 E-14$ & $\mathrm{~F}$ & $-2.77 \mathrm{E}-14$ \\
\hline & & u & $-1.85 \mathrm{E}-12$ & U & $-7.47 E-13$ & U & $-9.36 \mathrm{E}-13$ & U & $-3.49 E-13$ & U & $-2.31 E-13$ \\
\hline & & If & 2.9 & If & 2.9 & If & 2.9 & If & 2.9 & If & 2.9 \\
\hline & & $\theta$ & 128.39 & $\theta$ & 25.68 & $\theta$ & 2.85 & $\theta$ & 8.56 & $\theta$ & 5.14 \\
\hline & & $\mathrm{ff}+\theta$ & 131.29 & $\mathrm{rf}+\theta$ & 28.58 & $\mathrm{rf}+\theta$ & 5.75 & If $+\theta$ & 11.46 & If $+\theta$ & 8.04 \\
\hline & & $\tau$ & $-1.57 E-12$ & $\tau$ & $-4.37 E-12$ & $\tau$ & $-7.08 E-13$ & $\tau$ & $-7.61 E-13$ & $\tau$ & $-2.23 E-13$ \\
\hline & & tg & 1.18 & $\operatorname{tg}$ & 0.17 & $\operatorname{tg}$ & 1.32 & $\operatorname{tg}$ & 0.46 & $\operatorname{tg}$ & 1.04 \\
\hline & & arctg & 0.87 & $\operatorname{arctg}$ & 0.17 & $\operatorname{arctg}$ & 0.92 & $\operatorname{arctg}$ & 0.43 & $\operatorname{arctg}$ & 0.80 \\
\hline & & degrees $^{\circ}$ & 49.75 & degrees ${ }^{\circ}$ & 9.71 & degrees $^{\circ}$ & 52.94 & degrees $^{\circ}$ & 24.63 & degrees $^{\circ}$ & 46.02 \\
\hline
\end{tabular}

\begin{tabular}{|c|c|c|c|c|c|c|c|c|c|c|c|c|c|c|c|c|c|}
\hline \multicolumn{18}{|l|}{ SER22 } \\
\hline & $\phi^{\circ}$ & & & & & & & & & & & & & & & & \\
\hline CALCULATED & -42 & $\phi^{\circ}$ & & & & & & & & & & & & & & & \\
\hline \multirow{14}{*}{ EXPERMENTAL } & -47 & Electrostatic Inter & & Hydrophobicint & ration & Hydrophobicinter & & Hydrophobicinter & & H-bond & & H.bond & & H-bond & & Hydrophobicinter & \\
\hline & & between terminal & harres & & & & & & & & & & & & & & \\
\hline & & $q G \mid Y: N$ & 1 & ASER22:HB1 & 0.035 & qGIY20:CA & $\cdot 0.0252$ & ASER2:HB1 & 0.0352 & QSER22:0G & $\cdot 0.0546$ & ASER22:0G & $\cdot 0.6546$ & QSERR2Z:OG & $\cdot 0.0546$ & qulle24:CG2 & .0 .043 \\
\hline & & qASNM3:0 & -1 & qALA21:CB & -0.1825 & ASER2:HB2 & 0.035 & qIEN19:CO1 & -0.4121 & qHIS33:HE2 & 0.3649 & ASER25:HG1 & 0.4275 & q145266:H21 & 0.34 & ASER2:HB2 & 0.035 \\
\hline & & ii & 154.93 & ii & 5.25 & i் & 6.97 & ii & 11.43 & ri & 7.22 & ri & 12.3 & ri & 10.66 & ii & 8.03 \\
\hline & & $\mathrm{F}$ & $-1.195-14$ & $\mathrm{~F}$ & $-6.645-14$ & $\mathrm{~F}$ & $-5.21 E-15$ & $\mathrm{~F}$ & $-3.18 E-14$ & $\mathrm{~F}$ & $-131 E-12$ & $\mathrm{~F}$ & $-5.30 E-13$ & $\mathrm{~F}$ & $-5.62 E-13$ & $\mathrm{~F}$ & $-6.69 \mathrm{E}-15$ \\
\hline & & U & $-1.85 E-12$ & U & $-3.496-13$ & U & $-3.635-14$ & U & $-3.646-13$ & U & $-9.495-12$ & U & $-6.525-12$ & U & $-5.996-12$ & U & $-5.375-14$ \\
\hline & & If & 2.9 & if & 2.9 & if & 2.9 & If & 2.9 & if & 2.9 & if & 2.9 & if & 2.9 & if & 2.9 \\
\hline & & $\theta$ & 17.12 & $\theta$ & 8.56 & $\theta$ & 25.68 & $\theta$ & 285 & $\theta$ & 17.12 & $\theta$ & 17.12 & $\theta$ & 17.12 & $\theta$ & 2.85 \\
\hline & & $i+\theta$ & 20.02 & $f+\theta$ & 11.46 & $n+\theta$ & 28.58 & $\mathrm{f}+\theta$ & 5.75 & $\mathrm{r}+\theta$ & 20.02 & $\mathrm{r}+\theta$ & 20.02 & $n+\theta$ & 20.02 & $n+\theta$ & 5.75 \\
\hline & & $\tau$ & $-239 E-13$ & $\tau$ & $-7.61 E-13$ & $\tau$ & $-1.49 E-13$ & 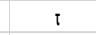 & $-1.88 E-13$ & $\tau$ & $-2.63 E-11$ & 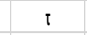 & $-1.06 E-11$ & $\tau$ & $-1.125-11$ & 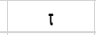 & $-3.855-14$ \\
\hline & & $\operatorname{tg}$ & 7.74 & $\operatorname{tg}$ & 0.46 & $\operatorname{tg}$ & 0.24 & $\operatorname{tg}$ & 1.99 & $\operatorname{tg}$ & 0.36 & $\operatorname{tg}$ & 0.61 & $\operatorname{tg}$ & 0.53 & $\operatorname{tg}$ & 1.40 \\
\hline & & arteg & 1.4 & artg & 0.43 & artg & 0.24 & artg & 1.10 & $\operatorname{arctg}$ & 0.35 & artg & 0.55 & artg & 0.49 & artg & 0.95 \\
\hline & & degrees ${ }^{\circ}$ & 82.68 & degrees ${ }^{\circ}$ & 24.63 & degrees $s^{\circ}$ & 13.71 & degrees $s^{\circ}$ & 63.31 & degrees ${ }^{\circ}$ & 19.84 & degrees" & 31.58 & degrees ${ }^{\circ}$ & 28.05 & degrees $^{\circ}$ & 54.41 \\
\hline
\end{tabular}

\begin{tabular}{|c|c|c|c|c|c|c|c|c|c|c|c|c|c|c|c|}
\hline \multicolumn{16}{|l|}{ HIS23 } \\
\hline & $\phi^{\circ}$ & & & & & & & & & & & & & & \\
\hline \multirow{15}{*}{$\begin{array}{l}\text { CALCULATED } \\
\text { EXPERIMENTLL }\end{array}$} & -68 & $\phi^{\circ}$ & & & & & & & & & & & & & \\
\hline & .68 & Electrostatic Inter & & H-bond & & Hydrophobic inter & & Hydrophobicinter & & H-bond & & H-bond & & Hydrophobic int & action \\
\hline & & between termina & arres & & & & & & & & & & & & \\
\hline & & qGLY:N & 1 & QSER22:0G & -0.6546 & qHIIS23:HB1 & 0.0367 & qHIIS23:HB2 & 0.0367 & QSER25:HG1 & 0.4275 & qLYS26:HZ3 & 0.34 & qHIS23:HB2 & 0.0367 \\
\hline & & qASNA3:0 & -1 & qHIS23:HE2 & 0.3649 & qALA21:CB & -0.1825 & qIEU19:CD1 & -0.4121 & qHIS23:ND & -0.5727 & qHIS23:ND & .0 .5277 & qllle24:CG2 & -0.043 \\
\hline & & ri & 154.93 & ri & 7.22 & ri & 8.32 & ri & 13.73 & ri & 9.43 & $\dot{\mathrm{n}}$ & 14.41 & in & 6.87 \\
\hline & & $\mathrm{F}$ & $-1.19 E-14$ & $\mathrm{~F}$ & $-1,31 E-12$ & $\mathrm{~F}$ & $-2.77 E-14$ & $\mathrm{~F}$ & $-2,30 E-14$ & $\mathrm{~F}$ & $-7.898-13$ & $\mathrm{~F}$ & $-2.69 \mathrm{E}-13$ & $\mathrm{~F}$ & $-9.59 E-15$ \\
\hline & & u & $-1.85 E-12$ & u & $-9.49 E-12$ & u & $-2.31 E-13$ & u & $-3.166-13$ & u & $-7.44 E-12$ & $u$ & $-3.87 E-12$ & u & $-6.59 E-14$ \\
\hline & & if & 2.9 & if & 2.9 & if & 2.9 & if & 2.9 & if & 2.9 & if & 2.9 & if & 2.9 \\
\hline & & $\theta$ & 17.12 & $\theta$ & 17.12 & $\theta$ & 14.27 & $\theta$ & 4.76 & $\theta$ & 17.12 & $\theta$ & 17.12 & $\theta$ & 4.76 \\
\hline & & $\mathrm{ft}+\theta$ & 20.02 & $n+\theta$ & 20.02 & $\mathrm{r}+\theta$ & 17.17 & $f+\theta$ & 7.66 & $f+\theta$ & 20.02 & $\mathrm{ff}+\theta$ & 20.02 & $\mathrm{r}+\theta$ & 7.66 \\
\hline & & $\tau$ & $-2,39 \mathrm{E}-13$ & t & $-2.63 E-11$ & $\tau$ & $-4.76 E-13$ & $\tau$ & $-1.766-13$ & $\tau$ & $-1.588-11$ & $\tau$ & $-5.38 E-12$ & $\tau$ & $-7.34 E-14$ \\
\hline & & tg & 7.74 & $\operatorname{tg}$ & 0.36 & $\operatorname{tg}$ & 0.48 & tg & 1.79 & $\operatorname{tg}$ & 0.47 & $\operatorname{tg}$ & 0.72 & $\operatorname{tg}$ & 0.90 \\
\hline & & arttg & 1.44 & arctg & 0.35 & arctg & 0.45 & arctg & 1.06 & artg & 0.44 & arctg & 0.62 & arctg & 0.73 \\
\hline & & degrees $^{\circ}$ & 82.68 & degrees ${ }^{\circ}$ & 19.84 & degrees $^{\circ}$ & 25.87 & degrees $^{\circ}$ & 60.89 & degrees $^{\circ}$ & 25.24 & degrees $^{\circ}$ & 35.76 & degrees ${ }^{\circ}$ & 41.93 \\
\hline
\end{tabular}

\begin{tabular}{|c|c|c|c|c|c|c|c|c|c|c|c|c|c|c|c|c|c|c|c|}
\hline \multicolumn{20}{|l|}{ ILE24 } \\
\hline & $\phi^{\circ}$ & & & & & & & & & & & & & & & & & & \\
\hline \multirow{15}{*}{$\begin{array}{l}\text { CALCULATED } \\
\text { EXPERMENTAL }\end{array}$} & -68 & $\phi^{\circ}$ & & & & & & & & & & & & & & & & & \\
\hline & -68 & \multirow{2}{*}{\multicolumn{2}{|c|}{$\begin{array}{l}\text { Electrostatic Interction } \\
\text { between terminal charges }\end{array}$}} & \multicolumn{2}{|c|}{ Hydrophobicinteraction } & \multicolumn{2}{|c|}{ Hydrophobicinteraction } & \multicolumn{2}{|c|}{ Hydrophobicinteraction } & \multicolumn{2}{|c|}{ Hydrophobicinteraction } & \multicolumn{2}{|c|}{ Hydrophobicinteraction } & \multicolumn{2}{|c|}{ Hydrophobicinteraction } & \multicolumn{2}{|c|}{ Hydrophobicinteraction } & \multicolumn{2}{|c|}{ Hydrophobicinteraction } \\
\hline & & & & & & & & & & & & & & & & & & & \\
\hline & & qGIY:N & 1 & qH1523:HB2 & 0.0367 & q\|E24:CG2 & 0.043 & qulle24:HG21 & 0.0186 & qGIY20:HA1 & 0.0698 & q\|l[E2:CG1 & $\cdot 0.043$ & q\|E24:CG1 & 0.043 & qulE2:CGI & -0.043 & qulE24:CG2 & $\cdot 0.043$ \\
\hline & & QASNM3:O & -1 & q11E24:G62 & -0.043 & QSER2R2:HB2 & 0.035 & QALA21:CB & -0.1825 & qlle24:CD & .0 .066 & QSER25:HB2 & 0.032 & q1Y526:HB12 & 0.032 & qTHR27:H621 & 0.0642 & qMEE28:HB2 & 0.0241 \\
\hline & & $\dot{n}$ & 154.93 & i & 6.87 & i & 8.03 & ก் & 1256 & ii & 12.25 & ri & 6.12 & กi & 9.17 & กี & 12.65 & ii & 14 \\
\hline & & $\mathrm{F}$ & $-1.195-14$ & $\mathrm{~F}$ & $-9.59-15$ & $\mathrm{~F}$ & $.6695 \cdot 15$ & $\mathrm{~F}$ & $.6 .17=-15$ & $\mathrm{~F}$ & $.8800-15$ & $\mathrm{f}$ & $-1.166-14$ & $\mathrm{~F}$ & $-5.315-15$ & $\mathrm{~F}$ & $-4.955-15$ & $\mathrm{~F}$ & $.1 .525-15$ \\
\hline & & u & $-1.85-12$ & u & $.6595-14$ & u & $.5376: 14$ & u & $.7 .75 E-14$ & u & $-1.085-13$ & u & $-7.095-14$ & u & $-4.87=-14$ & u & $-6.66-14$ & u & $-2,12 E-14$ \\
\hline & & if & 2.9 & if & 2.9 & if & 29 & if & 29 & if & 2.9 & if & 2.9 & if & 2.9 & if & 2.9 & if & 29 \\
\hline & & $\theta$ & 0.86 & $\theta$ & 15.41 & $\theta$ & 25.68 & $\theta$ & 25.68 & $\theta$ & 77.04 & $\theta$ & 25.68 & $\theta$ & 7.00 & $\theta$ & 15.41 & $\theta$ & 1101 \\
\hline & & $\mathrm{r}+\theta$ & 3.76 & $\mathrm{r}+\theta$ & 18.31 & $\mathrm{n}+\theta$ & 28.58 & $n+\theta$ & 2858 & $\mathrm{r}+\theta$ & 79.94 & $n+\theta$ & 28.58 & $\mathrm{nt}+\theta$ & 9.90 & $n+\theta$ & 1831 & $\mathrm{f}+\theta$ & 13.91 \\
\hline & & t & $-4.495-14$ & $\tau$ & $\cdot 1766-13$ & $\tau$ & -1915-13 & t & $\cdot 1.76 \cdot-13$ & $i$ & $-7.045-13$ & t & $.3 .315-13$ & t & $.5 .66-14$ & $\tau$ & $-9.066-14$ & $\tau$ & $\cdot 2.11 E-14$ \\
\hline & & $\operatorname{tg}$ & 44.25 & $\operatorname{tg}$ & 0.38 & $\operatorname{tg}$ & 0.28 & $\operatorname{tg}$ & 0.44 & tg & 0.15 & $\operatorname{tg}$ & 0.21 & $\operatorname{tg}$ & 0.93 & $\operatorname{tg}$ & 0.69 & $\operatorname{tg}$ & 101 \\
\hline & & artig & 1.55 & artg & 0.36 & artig & 0.27 & artig & 0.41 & arttg & 0.15 & artg & 0.21 & artig & 0.75 & artig & 0.60 & artig & 0.79 \\
\hline & & degrees $^{\circ}$ & 88.66 & degrees $^{\circ}$ & 20.58 & degrees & 15.70 & degrees & 23.74 & degrees ${ }^{\circ}$ & 8.72 & degrees & 12.09 & degrees ${ }^{\circ}$ & 42.82 & degrees & 34.66 & degrees & 45.22 \\
\hline
\end{tabular}



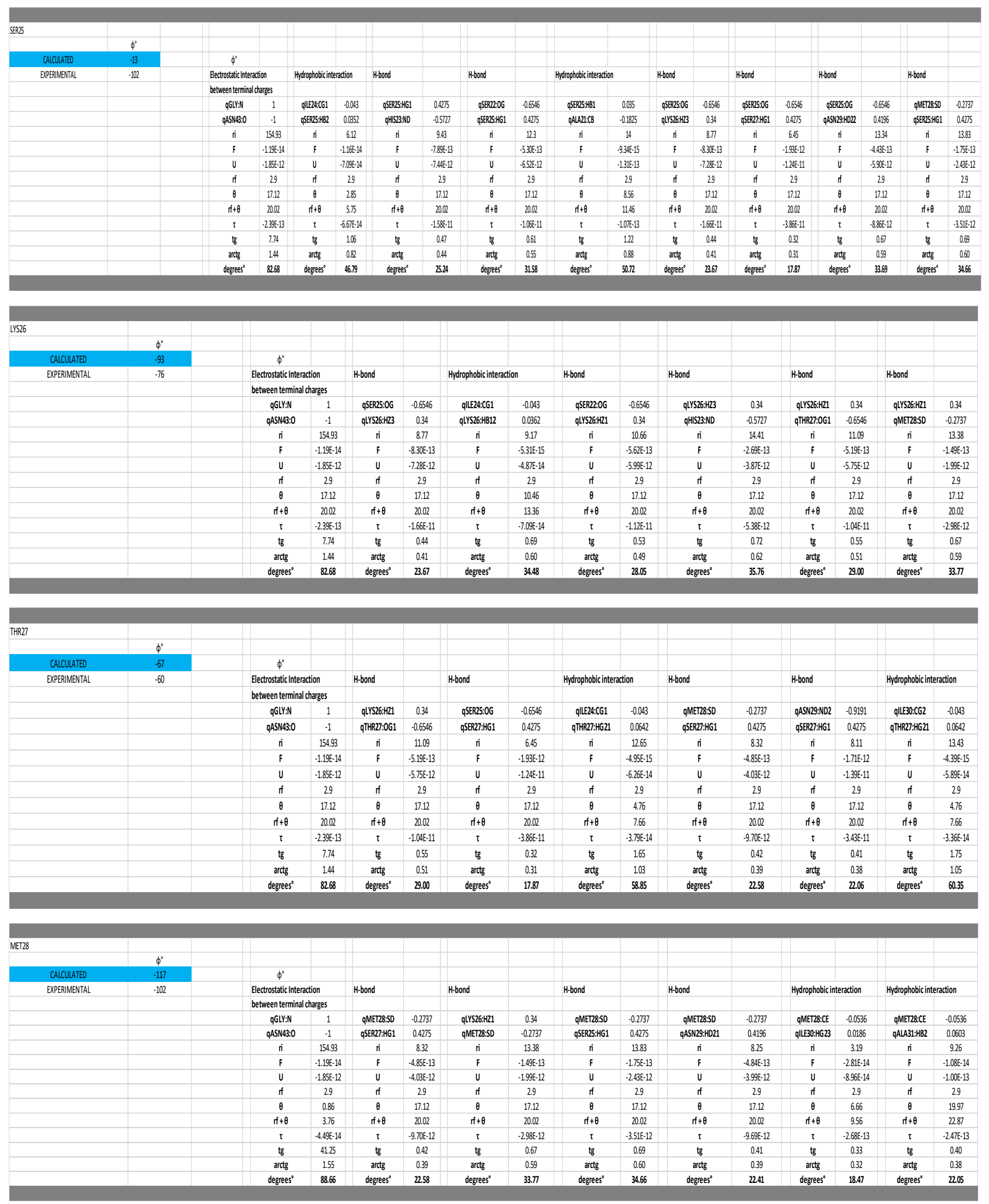

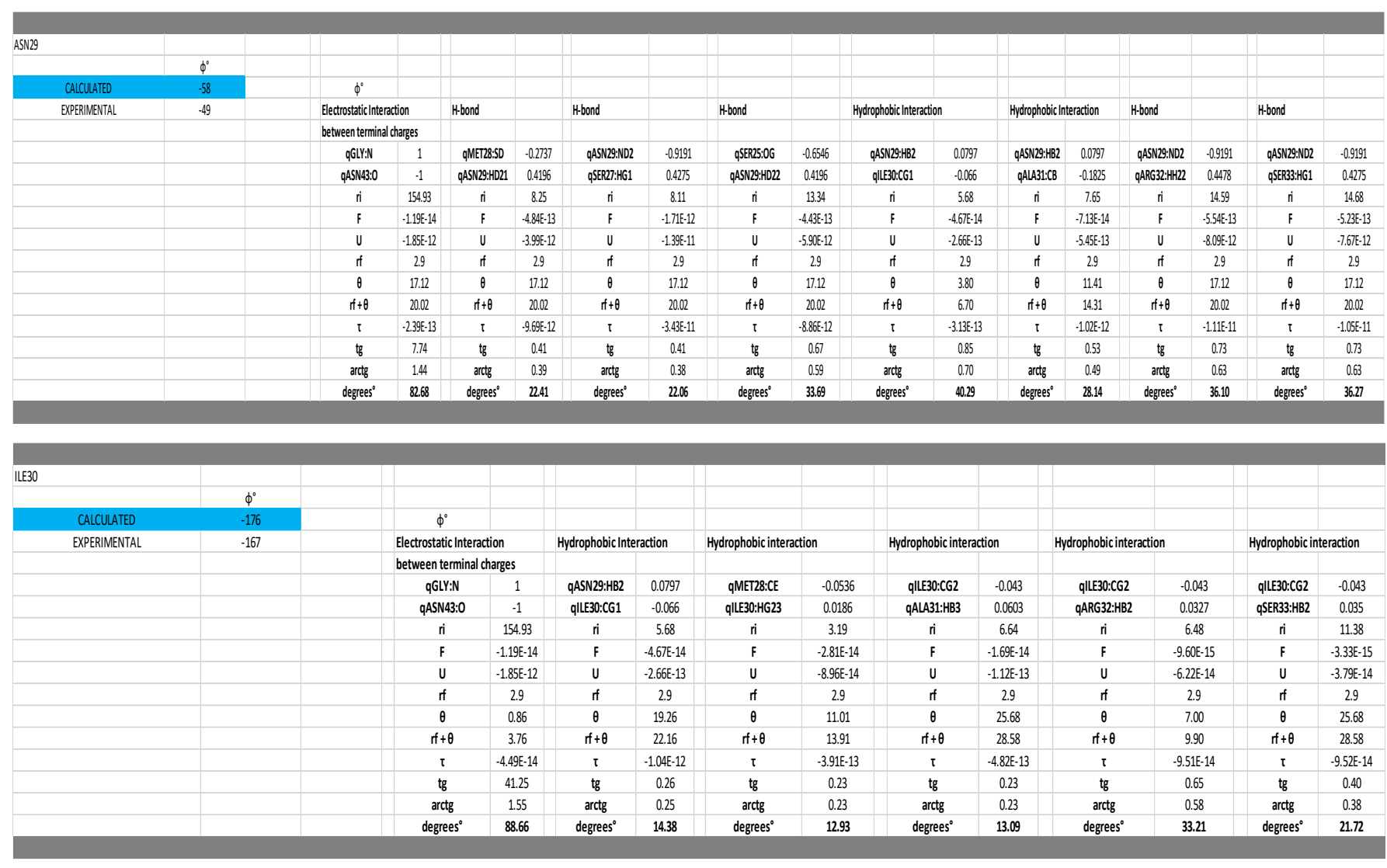

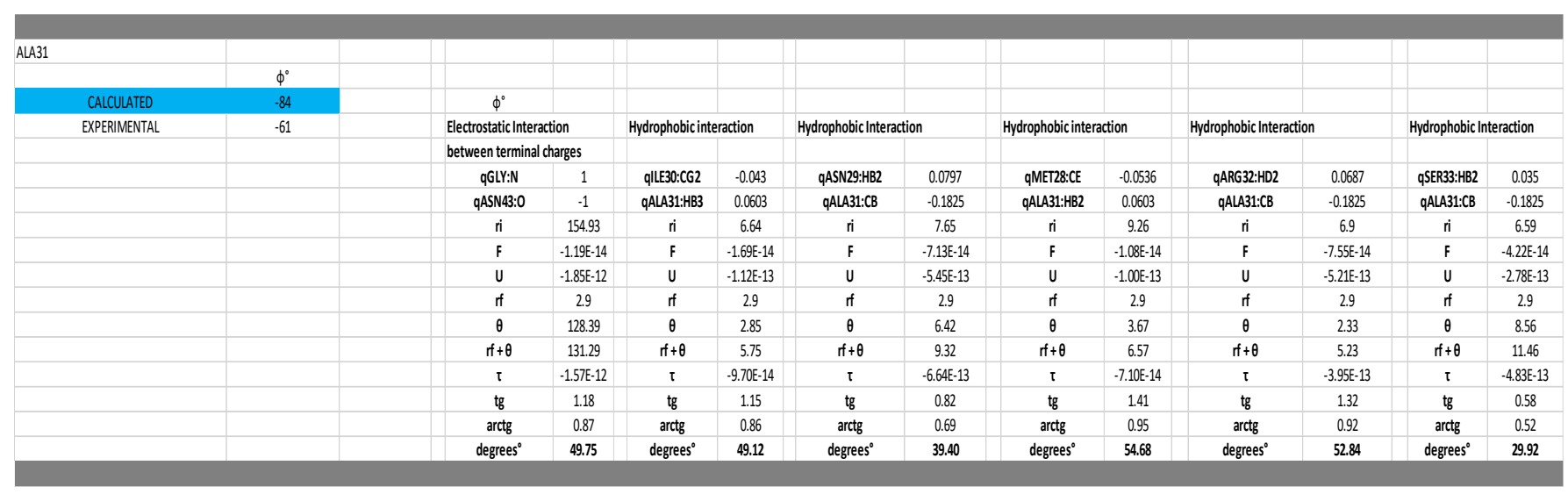

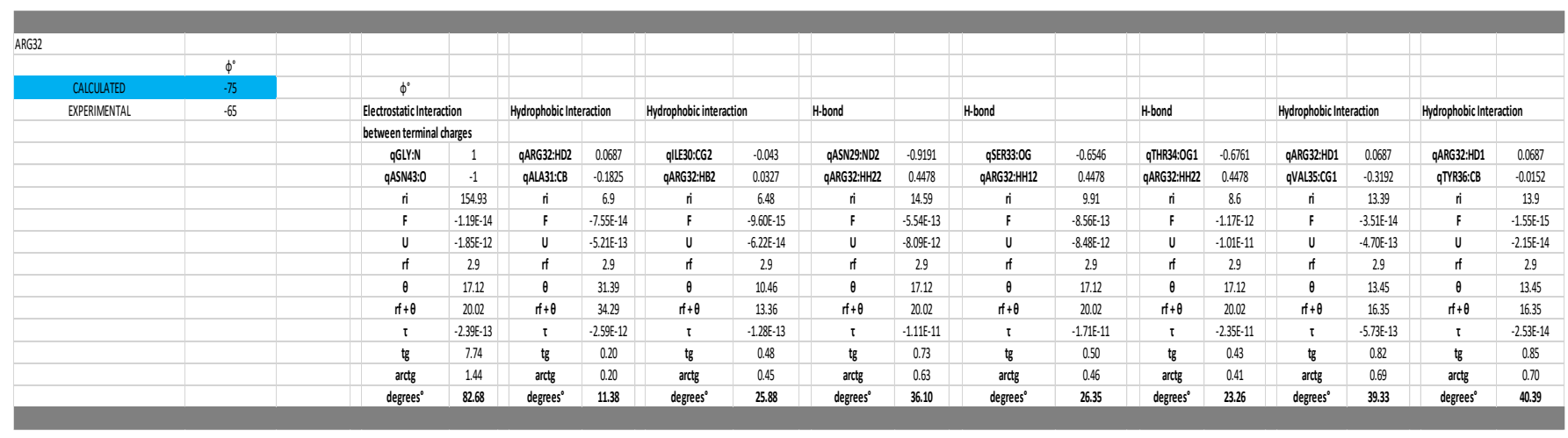



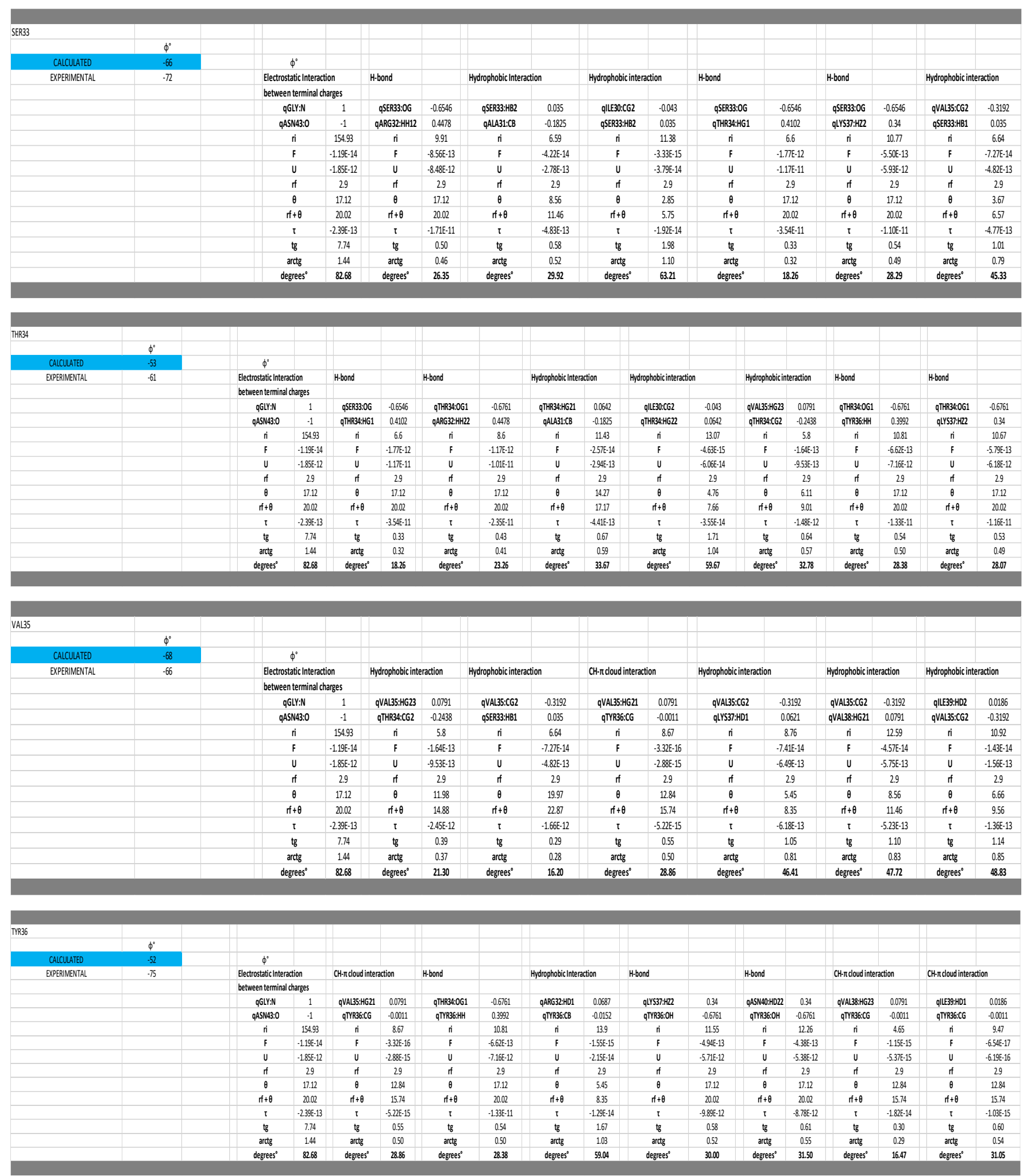


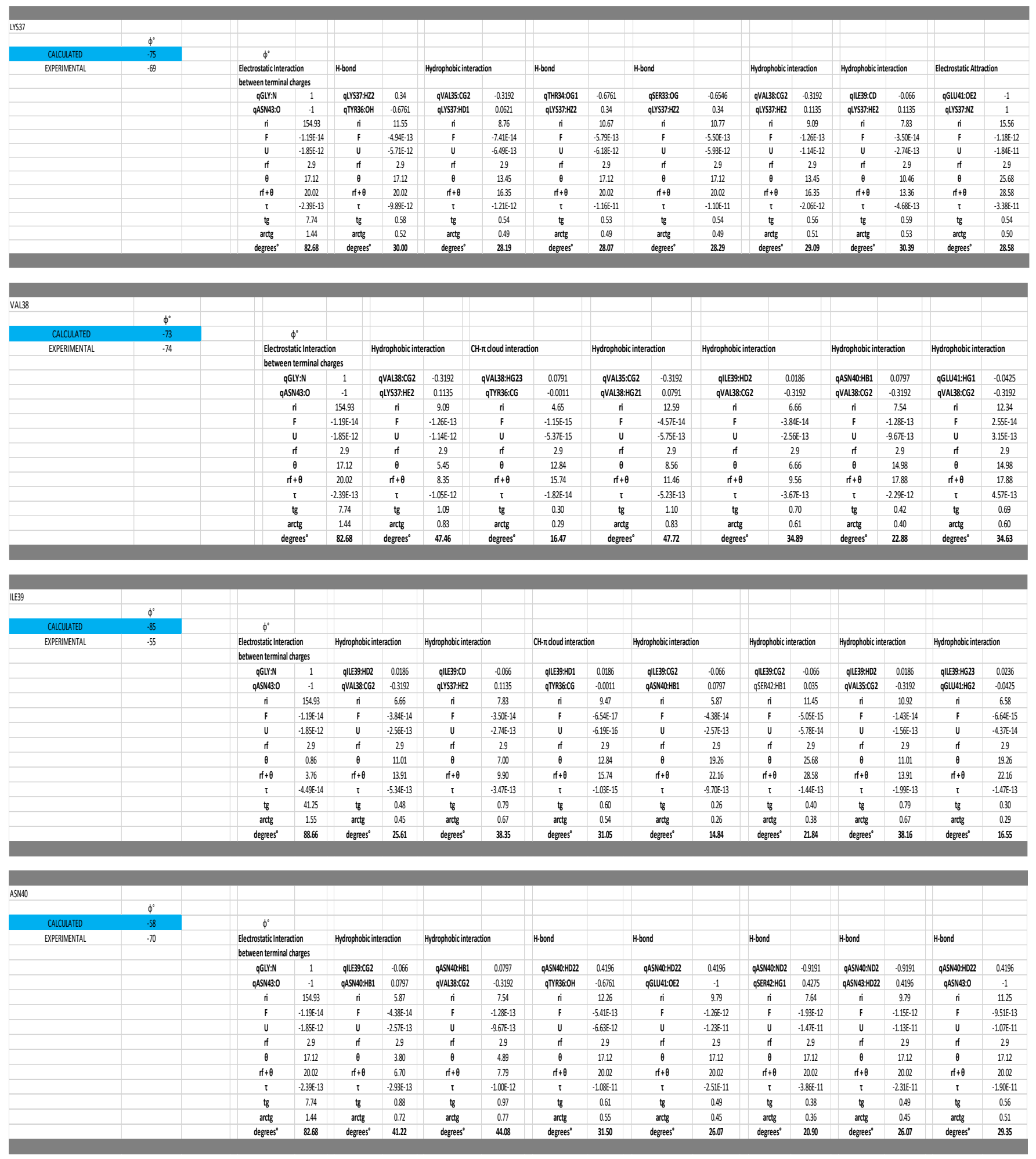



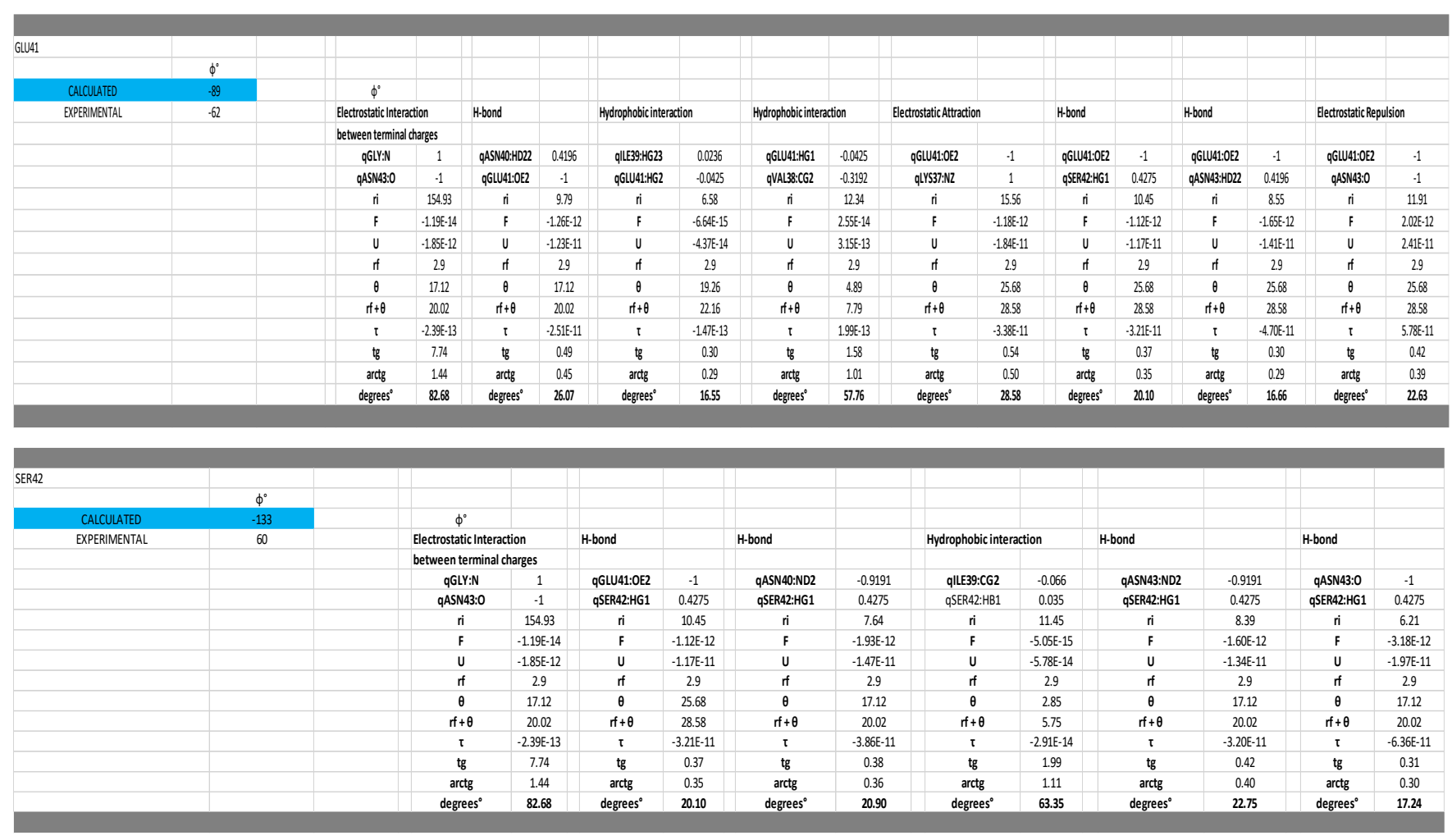

\begin{tabular}{|c|c|c|c|c|c|c|c|c|c|c|c|c|c|c|c|c|c|}
\hline \multicolumn{18}{|l|}{ ANN43 } \\
\hline & $\phi^{\circ}$ & & & & & & & & & & & & & & & & \\
\hline \multirow{14}{*}{$\begin{array}{l}\text { CALCULATED } \\
\text { EXPERIMENTAL }\end{array}$} & .79 & $\phi^{\circ}$ & & & & & & & & & & & & & & & \\
\hline & .79 & Electrostatic Inte & & H.bond & & H.bond & & H-bond & & Electrostatic Repulsio & & H.bond & & H.bond & & Hydrophobicinter & \\
\hline & & between termina & & & & & & & & & & & & & & & \\
\hline & & $q G \mid Y: N$ & 1 & qASN43:ND2 & -0.9191 & qASN43:0 & -1 & qGLU41:062 & -1 & qGLU41:0E2 & -1 & qASN40:ND2 & -0.9191 & qASN40:HD22 & 0.4196 & qIll39:CG2 & -0.066 \\
\hline & & gASNA3:0 & -1 & GSERA2:HG1 & 0.4275 & QSERA2:HG1 & 0.4275 & gASNA3:H022 & 0.4196 & qASNA3:0 & -1 & gASNA3:H022 & 0.4196 & gASNA3:O & -1 & qASNA3:HB1 & 0.0797 \\
\hline & & ri & 154.93 & กi & 8.39 & กi & 6.21 & ri & 8.55 & ri & 11.91 & ri & 9.79 & ii & 11.25 & i & 13.57 \\
\hline & & $\mathrm{F}$ & $-1.199-14$ & $\mathrm{~F}$ & $-1.600-12$ & $\mathrm{~F}$ & $-3.188-12$ & $\mathrm{~F}$ & $-1.655-12$ & $\mathrm{~F}$ & $2.022-12$ & $\mathrm{~F}$ & $-1.155-12$ & $\mathrm{~F}$ & $-9.511-13$ & $\mathrm{~F}$ & $8.199-15$ \\
\hline & & u & $-1.855-12$ & u & $-1.344-11$ & u & $-1.97-11$ & u & $-1.41 E-11$ & u & 2.41E-11 & u & $-1.135-11$ & u & $-1077-11$ & $u$ & $-1.111-13$ \\
\hline & & if & 2.9 & if & 2.9 & if & 2.9 & if & 2.9 & if & 2.9 & if & 2.9 & if & 2.9 & if & 2.9 \\
\hline & & $\mathrm{r}+\theta$ & 20.02 & $\mathrm{r}+\theta$ & 20.02 & $r+\theta$ & 20.02 & $\mathrm{r}+\theta$ & 28.58 & $r+\theta$ & 28.58 & $\mathrm{r}+\mathrm{\theta}$ & 20.02 & $r+\theta$ & 20.02 & $r+\theta$ & 6.70 \\
\hline & & $\tau$ & $-2.395-13$ & $\tau$ & $-3.200-11$ & $\tau$ & $-6.366-11$ & $\tau$ & $-4,70-11$ & $\tau$ & $5.788-11$ & $\tau$ & $-2,311-11$ & $\tau$ & $-1.906-11$ & $\tau$ & $-5.49-14$ \\
\hline & & $\operatorname{tg}$ & 7.74 & $\operatorname{tg}$ & 0.42 & $\operatorname{tg}$ & 0.31 & $\operatorname{tg}$ & 0.30 & $\operatorname{tg}$ & 0.42 & $\operatorname{tg}$ & 0.49 & $\operatorname{tg}$ & 0.56 & $\operatorname{tg}$ & 2.02 \\
\hline & & artg & 1.4 & artg & 0.40 & artg & 0.30 & arttg & 0.29 & artc & 0.39 & arctg & 0.45 & artg & 0.51 & artg & 1.11 \\
\hline & & degrees $^{\circ}$ & 82.68 & degrees $^{\circ}$ & 22.75 & degreses $^{\circ}$ & 17.24 & degrees $^{\circ}$ & 16.66 & degrees ${ }^{\circ}$ & 22.63 & degrees $^{\circ}$ & 26.07 & degrees $^{\circ}$ & 29.35 & degrees $^{\circ}$ & 63.74 \\
\hline
\end{tabular}


